\title{
Generic representations of orthogonal groups: projective functors in the category $\mathcal{F}_{\text {quad }}$
}

by

\author{
Christine Vespa (Strasbourg)
}

\begin{abstract}
We continue the study of the category of functors $\mathcal{F}_{\text {quad }}$, associated to $\mathbb{F}_{2}$ vector spaces equipped with a nondegenerate quadratic form, initiated in J. Pure Appl. Algebra 212 (2008) and Algebr. Geom. Topology 7 (2007). We define a filtration of the standard projective objects in $\mathcal{F}_{\text {quad }}$; this refines to give a decomposition into indecomposable factors of the first two standard projective objects in $\mathcal{F}_{\text {quad }}: P_{H_{0}}$ and $P_{H_{1}}$. As an application of these two decompositions, we give a complete description of the polynomial functors in $\mathcal{F}_{\text {quad }}$.
\end{abstract}

Introduction. In [12] we defined the category of functors $\mathcal{F}_{\text {quad }}$ from a category having as objects the nondegenerate $\mathbb{F}_{2}$-quadratic spaces to the category $\mathcal{E}$ of $\mathbb{F}_{2}$-vector spaces, where $\mathbb{F}_{2}$ is the field with two elements. The motivation for the construction of this category is to obtain, for orthogonal groups over $\mathbb{F}_{2}$, an analogous framework to that which exists for the general linear groups. We recall that the category $\mathcal{F}$ of functors from the category $\mathcal{E}^{f}$ of finite-dimensional $\mathbb{F}_{2}$-vector spaces to the category $\mathcal{E}$ of all $\mathbb{F}_{2}$-vector spaces is a very useful tool for the study of the stable cohomology of general linear groups with suitable coefficients (see [4]). Another motivation, in topology, for the study of the category $\mathcal{F}$ is the connection between this category and unstable modules over the Steenrod algebra (see [9]).

In order to have a good understanding of the category $\mathcal{F}_{\text {quad }}$, we seek to classify its simple objects. We constructed in [12] two families of simple objects in $\mathcal{F}_{\text {quad }}$. The first one is obtained from the fully faithful, exact functor $\iota: \mathcal{F} \rightarrow \mathcal{F}_{\text {quad }}$, defined in [12], which preserves simple objects. By [6], the simple objects in $\mathcal{F}$ are in one-to-one correspondence with the irreducible representations of finite general linear groups over $\mathbb{F}_{2}$. The second

2000 Mathematics Subject Classification: 18A25, 20J99, 16D90, 20C20.

Key words and phrases: functor categories, quadratic forms over $\mathbb{F}_{2}$, Mackey functors, representations of orthogonal groups over $\mathbb{F}_{2}$. 
family is obtained from the fully faithful, exact functor $\kappa: \mathcal{F}_{\text {iso }} \rightarrow \mathcal{F}_{\text {quad }}$, which preserves simple objects, where $\mathcal{F}_{\text {iso }}$ is equivalent to the product of the categories of modules over the orthogonal groups of possibly degenerate quadratic forms. In [11], we constructed two families of simple objects in the category $\mathcal{F}_{\text {quad }}$ which are neither in the image of $\iota$ nor in the image of $\kappa$. These simple objects are subfunctors of the tensor product between an object in the image of $\iota$ and an object in the image of $\kappa$. We proved that these simple objects in $\mathcal{F}_{\text {quad }}$ are the composition factors of two particular mixed functors, defined in [11].

The aim of this paper is to begin a programme to obtain a complete classification of the simple objects in $\mathcal{F}_{\text {quad }}$. Accordingly, we seek to decompose the projective generators of this category into indecomposable factors and to obtain the simple factors of these indecomposable factors. This paper begins the study of the standard projective objects in the category $\mathcal{F}_{\text {quad }}$. Although explicit decompositions of all projective generators are not provided in this paper, we give several useful tools, results and examples for the realization of this programme. Furthermore, we deduce several interesting consequences for the structure of the category $\mathcal{F}_{\text {quad }}$. In work in progress, we obtain a general decomposition of a standard projective object $P_{H}$ of $\mathcal{F}_{\text {quad }}$ which is indexed by the subspaces of $H$. Here we present explicit decompositions of the standard projective objects associated to "small" quadratic spaces, since these decompositions play a fundamental rôle in the category $\mathcal{F}_{\text {quad }}$ (for example, for the description of the polynomial functors in $\mathcal{F}_{\text {quad }}$ ). Furthermore, recall that the decomposition of the standard injective $I_{\mathbb{F}_{2}}^{\mathcal{F}}$ of the category $\mathcal{F}$ and thus, by duality, that of the standard projective $P_{\mathbb{F}_{2}}^{\mathcal{F}}$, is fundamental for the comprehension of the other standard injectives of $\mathcal{F}$. Hence, the decompositions of the two smaller standard projectives of $\mathcal{F}_{\text {quad }}$ represent an important step in the understanding of the category $\mathcal{F}_{\text {quad }}$.

We briefly summarize the contents of this paper. After some recollections on the category $\mathcal{F}_{\text {quad }}$, where we recall the definitions of the isotropic functors and mixed functors, we define a filtration of the standard projective objects $P_{V}$ in $\mathcal{F}_{\text {quad: }}$ :

$$
0 \subset P_{V}^{(0)} \subset P_{V}^{(1)} \subset \cdots \subset P_{V}^{(\operatorname{dim}(V)-1)} \subset P_{V}^{(\operatorname{dim}(V))}=P_{V} .
$$

We obtain a general description of the two extremities of this filtration.

Theorem. Let $V$ be a nondegenerate $\mathbb{F}_{2}$-quadratic space.

(1) There is a natural equivalence $P_{V}^{(0)} \simeq \iota\left(P_{\epsilon(V)}^{\mathcal{F}}\right)$, where $\iota: \mathcal{F} \rightarrow \mathcal{F}_{\text {quad }}$, $\epsilon$ is the functor that forgets the quadratic form, and $P_{\epsilon(V)}^{\mathcal{F}}$ is the standard projective object in $\mathcal{F}$ associated to the vector space $\epsilon(V)$.

(2) The functor $P_{V}^{(0)}$ is a direct summand of $P_{V}$. 
Proposition. Let $V$ be a nondegenerate $\mathbb{F}_{2}$-quadratic space. We have a natural equivalence

$$
P_{V} / P_{V}^{(\operatorname{dim}(V)-1)} \simeq \kappa\left(\text { iso }_{V}\right),
$$

where $\kappa: \mathcal{F}_{\text {iso }} \rightarrow \mathcal{F}_{\text {quad }}$ and iso $V$ is an isotropic functor in $\mathcal{F}_{\text {iso. }}$.

An important consequence concerning the functor $P_{V}^{(0)}$ is the following result.

TheOREM. The category $\iota(\mathcal{F})$ is a thick subcategory of $\mathcal{F}_{\text {quad }}$.

Then by an explicit study of the filtration of the functors $P_{H_{0}}$ and $P_{H_{1}}$ we obtain the following fundamental decompositions of these two standard projective functors.

THEOREM.

(1) The standard projective object $P_{H_{0}}$ admits the decomposition

$$
P_{H_{0}}=\iota\left(P_{\mathbb{F}_{2}}^{\mathcal{F}}\right) \oplus\left(\operatorname{Mix}_{0,1} \oplus 2 \oplus \operatorname{Mix}_{1,1}\right) \oplus \kappa\left(\text { iso }_{H_{0}}\right),
$$

where $\operatorname{Mix}_{0,1}$ and $\operatorname{Mix}_{1,1}$ are two mixed functors and iso $_{H_{0}}$ is an isotropic functor.

(2) The standard projective object $P_{H_{1}}$ admits the decomposition

$$
P_{H_{1}}=\iota\left(P_{\mathbb{F}_{2}{ }^{\oplus 2}}^{\mathcal{F}}\right) \oplus \operatorname{Mix}_{1,1} \oplus 3 \oplus \kappa\left(\text { iso }_{H_{1}}\right),
$$

where $\mathrm{Mix}_{1,1}$ is a mixed functor and iso $_{H_{1}}$ is an isotropic functor.

These decompositions have several interesting consequences. Firstly, thanks to this theorem we can complete the study of the functors Mix $\mathrm{M}_{0,1}$ and $\mathrm{Mix}_{1,1}$ started in [11] by the following result.

Proposition. The functors $\mathrm{Mix}_{0,1}$ and $\mathrm{Mix}_{1,1}$ are indecomposable.

We emphasize that the structure of the direct summands of $P_{H_{0}}$ and $P_{H_{1}}$ is completely understood. The structure of the isotropic functors is given in [12], that of the mixed functors $\operatorname{Mix}_{0,1}$ and $\operatorname{Mix}_{1,1}$ is the main result of [11] and is completed by the above proposition, and that of $P_{\mathbb{F}_{2}}^{\mathcal{F}}$ follows from [6]. Moreover, these decompositions give rise to a classification of the simple functors $S$ in $\mathcal{F}_{\text {quad }}$ such that $S\left(H_{0}\right) \neq 0$ or $S\left(H_{1}\right) \neq 0$.

Proposition. The isomorphism classes of nonconstant simple functors in $\mathcal{F}_{\text {quad }}$ such that either $S\left(H_{0}\right) \neq 0$ or $S\left(H_{1}\right) \neq 0$ are:

$$
\iota\left(\Lambda^{1}\right), \iota\left(\Lambda^{2}\right), \iota\left(S_{(2,1)}\right), \kappa\left(\operatorname{iso}_{(x, 0)}\right), \kappa\left(\operatorname{iso}_{(x, 1)}\right), R_{H_{0}}, R_{H_{1}}, S_{H_{1}},
$$

where $R_{H_{0}}, R_{H_{1}}$ and $S_{H_{1}}$ are the simple functors introduced in Corollary 1.7.

These decompositions also allow us to derive some homological calculations in the category $\mathcal{F}_{\text {quad }}$. 
Proposition. For any natural number $n$, we have

$$
\operatorname{Ext}_{\mathcal{F}_{\text {quad }}}^{n}\left(R_{H_{0}}, R_{H_{0}}\right) \simeq \mathbb{F}_{2} \text { and } \operatorname{Ext}_{\mathcal{F}_{\text {quad }}}^{n}\left(R_{H_{1}}, R_{H_{1}}\right) \simeq \mathbb{F}_{2},
$$
where $R_{H_{0}}$ and $R_{H_{1}}$ are the simple functors introduced in Corollary 1.7.

Finally, after having introduced the notion of polynomial functor for the category $\mathcal{F}_{\text {quad }}$, which generalizes that for $\mathcal{F}$, we obtain the following result as an application of the classification of the simple functors $S$ in $\mathcal{F}_{\text {quad }}$ such that $S\left(H_{0}\right) \neq 0$ or $S\left(H_{1}\right) \neq 0$ and of the thickness of the subcategory $\iota(\mathcal{F})$ of $\mathcal{F}_{\text {quad }}$.

TheOREM. The polynomial functors in $\mathcal{F}_{\text {quad }}$ are in the image of the functor $\iota: \mathcal{F} \rightarrow \mathcal{F}_{\text {quad }}$.

Most of the results of this paper are contained in the Ph.D. thesis of the author [10].

1. The category $\mathcal{F}_{\text {quad }}$ : some recollections. In this section we recall some definitions and results about the category $\mathcal{F}_{\text {quad }}$ obtained in [12].

Let $\mathcal{E}_{q}$ be the category having as objects finite-dimensional $\mathbb{F}_{2}$-vector spaces equipped with a nondegenerate quadratic form and with morphisms linear maps that preserve the quadratic forms. By the classification of quadratic forms over the field $\mathbb{F}_{2}$ (see, for instance, [7]) we know that only spaces of even dimension can be nondegenerate and, for a fixed even dimension, there are two inequivalent nondegenerate spaces, which are distinguished by the Arf invariant. We will denote by $H_{0}$ (resp. $H_{1}$ ) the nondegenerate quadratic space of dimension two such that $\operatorname{Arf}\left(H_{0}\right)=0$ (resp. $\left.\operatorname{Arf}\left(H_{1}\right)=1\right)$. The orthogonal sum of two nondegenerate quadratic spaces $\left(V, q_{V}\right)$ and $\left(W, q_{W}\right)$, denoted by $W \perp V$, is, by definition, the quadratic space $\left(V \oplus W, q_{V \oplus W}\right)$ where $q_{V \oplus W}(v, w)=q_{V}(v)+q_{W}(w)$. Recall that the spaces $H_{0} \perp H_{0}$ and $H_{1} \perp H_{1}$ are isomorphic. Observe that the morphisms of $\mathcal{E}_{q}$ are injective linear maps and this category does not admit push-outs or pull-backs. There exists a pseudo push-out in $\mathcal{E}_{q}$ that allows us to generalize the construction of the category of co-spans of Bénabou [1] and thus to define the category $\mathcal{T}_{q}$ in which there exist retractions.

Definition 1.1. The category $\mathcal{T}_{q}$ has as objects those of $\mathcal{E}_{q}$ and, for any objects $V$ and $W$ in $\mathcal{T}_{q}, \operatorname{Hom}_{\mathcal{T}_{q}}(V, W)$ is the set of equivalence classes of diagrams in $\mathcal{E}_{q}$ of the form $V \stackrel{f}{\rightarrow} X \stackrel{g}{\leftarrow} W$ for the equivalence relation generated by the relation $\mathcal{R}$ defined as follows:

$$
V \stackrel{f}{\rightarrow} X_{1} \stackrel{g}{\leftarrow} W \quad \mathcal{R} \quad V \stackrel{u}{\rightarrow} X_{2} \stackrel{v}{\leftarrow} W
$$

if there exists a morphism $\alpha$ of $\mathcal{E}_{q}$ such that $\alpha \circ f=u$ and $\alpha \circ g=v$. The composition is defined using the pseudo push-out. The morphism in 
$\operatorname{Hom}_{\mathcal{T}_{q}}(V, W)$ represented by the diagram $V \stackrel{f}{\rightarrow} X \stackrel{g}{\leftarrow} W$ will be denoted by $[V \stackrel{f}{\rightarrow} X \stackrel{g}{\leftarrow} W]$.

REMARK 1.2. Each morphism in $\operatorname{Hom}_{\mathcal{T}_{q}}(V, W)$ is represented by a diagram of the form $V \stackrel{g}{\rightarrow} W \perp W^{\prime} \stackrel{i_{W}}{\longleftarrow} W$, where $i_{W}$ is the canonical inclusion. In the following, we will use this representation without further comment.

By definition, $\mathcal{F}_{\text {quad }}$ is the category of functors from $\mathcal{T}_{q}$ to $\mathcal{E}$. Hence $\mathcal{F}_{\text {quad }}$ is abelian and has enough projective objects. By the Yoneda lemma, for any object $V$ of $\mathcal{T}_{q}$, the functor $P_{V}=\mathbb{F}_{2}\left[\operatorname{Hom}_{\mathcal{T}_{q}}(V,-)\right]$ is a projective object and there is a natural isomorphism $\operatorname{Hom}_{\mathcal{F}_{\text {quad }}}\left(P_{V}, F\right) \simeq F(V)$ for all objects $F$ of $\mathcal{F}_{\text {quad. }}$. The set of functors $\left\{P_{V} \mid V \in \mathcal{S}\right\}$, named the standard projective objects in $\mathcal{F}_{\text {quad }}$, is a set of projective generators of $\mathcal{F}_{\text {quad }}$, where $\mathcal{S}$ is a set of representatives of isometry classes of nondegenerate quadratic spaces. and

There is a forgetful functor $\epsilon: \mathcal{T}_{q} \rightarrow \mathcal{E}^{f}$ in $\mathcal{F}_{\text {quad }}$, defined by $\epsilon(V)=\mathcal{O}(V)$

$$
\epsilon\left(\left[V \stackrel{f}{\rightarrow} W \perp W^{\prime} \stackrel{g}{\leftarrow} W\right]\right)=p_{g} \circ \mathcal{O}(f),
$$

where $p_{g}$ is the orthogonal projection from $W \perp W^{\prime}$ to $W$ and $\mathcal{O}: \mathcal{E}_{q} \rightarrow \mathcal{E}^{f}$ is the functor which forgets the quadratic form. By the fullness of the functor $\epsilon$ and an argument of essential surjectivity, we obtain the following theorem.

TheOREM $1.3([12])$. There is a functor $\iota: \mathcal{F} \rightarrow \mathcal{F}_{\text {quad }}$ which is exact, fully faithful and preserves simple objects.

In order to define another subcategory of $\mathcal{F}_{\text {quad }}$, we consider the category $\mathcal{E}_{q}^{\text {deg }}$ having as objects finite-dimensional $\mathbb{F}_{2}$-vector spaces equipped with a (possibly degenerate) quadratic form and with morphisms injective linear maps which preserve the quadratic forms. The category $\mathcal{E}_{q}^{\text {deg }}$ admits pull-backs; consequently, the category of $\operatorname{spans} \operatorname{Sp}\left(\mathcal{E}_{q}^{\mathrm{deg}}\right)([1])$ is defined. By definition, $\mathcal{F}_{\text {iso }}$ is the category of functors from $\operatorname{Sp}\left(\mathcal{E}_{q}^{\text {deg }}\right)$ to $\mathcal{E}$. As in the case of $\mathcal{F}_{\text {quad }}$, the category $\mathcal{F}_{\text {iso }}$ is abelian and has enough projective objects: by the Yoneda lemma, for any object $V$ of $\operatorname{Sp}\left(\mathcal{E}_{q}^{\text {deg }}\right)$, the functor $Q_{V}=\mathbb{F}_{2}\left[\operatorname{Hom}_{\mathrm{Sp}\left(\mathcal{E}_{q}^{\mathrm{deg}}\right)}(V,-)\right]$ is a projective object in $\mathcal{F}_{\text {iso. }}$. We define a particular family of functors in $\mathcal{F}_{\text {iso }}$, the isotropic functors, which form a set of projective generators and injective cogenerators of $\mathcal{F}_{\text {iso. }}$. The category $\mathcal{F}_{\text {iso }}$ is related to $\mathcal{F}_{\text {quad }}$ by the following theorem.

TheOREM $1.4([12])$. There is a functor $\kappa: \mathcal{F}_{\text {iso }} \rightarrow \mathcal{F}_{\text {quad }}$ which is exact, fully faithful and preserves simple objects.

We obtain a classification of the simple objects of the category $\mathcal{F}_{\text {iso }}$ from the following theorem. 
TheOrem $1.5([12])$. There is a natural equivalence of categories

$$
\mathcal{F}_{\text {iso }} \simeq \prod_{V \in \mathcal{S}} \mathbb{F}_{2}[O(V)] \text {-mod, }
$$

where $\mathcal{S}$ is a set of representatives of isometry classes of quadratic spaces (possibly degenerate) and $O(V)$ is the orthogonal group.

The object of $\mathcal{F}_{\text {iso }}$ which corresponds, via this equivalence, to the module $\mathbb{F}_{2}[O(V)]$ is the isotropic functor iso $V$, defined in [12]. Recall that, as a vector space, $\operatorname{iso}_{V}(W)$ is isomorphic to the subspace of $Q_{V}(W)$ generated by the elements $[V \stackrel{\text { Id }}{\longleftarrow} V \rightarrow W]$.

A straightforward consequence of the classification of the simple objects of $\mathcal{F}_{\text {iso }}$ in Theorem 1.5 is in the following corollary. Recall that, by definition, an object $F$ of $\mathcal{F}_{\text {quad }}$ is finite if it has a finite composition series with simple subquotients.

COROLlary 1.6. The isotropic functors $\kappa\left(\right.$ iso $\left._{V}\right)$ are finite in the category $\mathcal{F}_{\text {quad }}$.

In Section 3, in order to obtain the classification of the simple objects $S$ of $\mathcal{F}_{\text {quad }}$ such that either $S\left(H_{0}\right) \neq 0$ or $S\left(H_{1}\right) \neq 0$, we need the composition factors of the isotropic functors associated to some small quadratic spaces. For $\alpha \in\{0,1\}$, let $(x, \alpha)$ be the degenerate quadratic space of dimension one generated by $x$ such that $q(x)=\alpha$. Since the orthogonal groups $O(x, 0)$ and $O(x, 1)$ are trivial and $O\left(H_{0}\right) \simeq \mathfrak{S}_{2}$ and $O\left(H_{1}\right) \simeq \mathfrak{S}_{3}$, we deduce from Theorems 1.5 and 1.4 the following corollary.

Corollary 1.7.

(1) The functors $\kappa\left(\operatorname{iso}_{(x, 0)}\right)$ and $\kappa\left(\operatorname{iso}_{(x, 1)}\right)$ are simple in $\mathcal{F}_{\text {quad }}$.

(2) The functor $\kappa\left(\right.$ iso $\left._{H_{0}}\right)$ is indecomposable. We have a nonsplit short exact sequence

$$
0 \rightarrow R_{H_{0}} \rightarrow \kappa\left(\text { iso }_{H_{0}}\right) \rightarrow R_{H_{0}} \rightarrow 0,
$$

where $R_{H_{0}}$ is the functor obtained from the trivial representation of $O\left(H_{0}\right)$.

(3) The functor $\kappa\left(\right.$ iso $\left._{H_{1}}\right)$ admits the decomposition

$$
\kappa\left(\text { iso }_{H_{1}}\right)=F_{H_{1}} \oplus\left(S_{H_{1}}\right)^{\oplus 2},
$$

where $S_{H_{1}}$ is the functor obtained from the natural representation of $O\left(H_{1}\right)$, and $F_{H_{1}}$ is an indecomposable functor for which we have a nonsplit short exact sequence

$$
0 \rightarrow R_{H_{1}} \rightarrow F_{H_{1}} \rightarrow R_{H_{1}} \rightarrow 0,
$$

where $R_{H_{1}}$ is the functor obtained from the trivial representation of $O\left(H_{1}\right)$. 
In [11], we define a new family of functors in $\mathcal{F}_{\text {quad }}$, named the mixed functors, and we decompose two particular functors of this family: the functors $\mathrm{Mix}_{0,1}$ and $\mathrm{Mix}_{1,1}$. We recall the following description of these functors.

Proposition 1.8 ([11]). For $\alpha \in\{0,1\}$, the functors $\operatorname{Mix}_{\alpha, 1}: \mathcal{T}_{q} \rightarrow \mathcal{E}$ are defined by

$$
\operatorname{Mix}_{\alpha, 1}(V)=\mathbb{F}_{2}\left[S_{V}\right]
$$

where $S_{V}=\left\{\left(v_{1}, v_{2}\right) \mid v_{1} \in V, v_{2} \in V, q\left(v_{1}+v_{2}\right)=\alpha, B\left(v_{1}, v_{2}\right)=1\right\}$ and

$$
\begin{aligned}
\operatorname{Mix}_{\alpha, 1}\left(\left[V \stackrel{f}{\rightarrow} W \perp L \stackrel{i_{W}}{\longleftarrow} W\right]\right)\left[\left(v_{1}, v_{2}\right)\right] & \\
& = \begin{cases}{\left[\left(p_{W} \circ f\left(v_{1}\right), p_{W} \circ f\left(v_{2}\right)\right)\right]} & \text { if } f\left(v_{1}+v_{2}\right) \in W, \\
0 & \text { otherwise, }\end{cases}
\end{aligned}
$$

where $p_{W}$ is the orthogonal projection.

In [11], for any positive integer $n$, we defined subfunctors $L_{\alpha}^{n}$ of $\iota\left(\Lambda^{n}\right) \otimes$ $\kappa\left(\operatorname{iso}_{(x, \alpha)}\right)$, where $\Lambda^{n}$ is the $n$th exterior power, and we proved that these functors are simple. The functor $L_{\alpha}^{1}$ is equivalent to $\kappa\left(\operatorname{iso}_{(x, \alpha)}\right)$. We obtain the following result.

Theorem 1.9 ([11]). Let $\alpha \in\{0,1\}$.

(1) The functor $\operatorname{Mix}_{\alpha, 1}$ is infinite.

(2) There exists a subfunctor $\Sigma_{\alpha, 1}$ of $\mathrm{Mix}_{\alpha, 1}$ such that we have a short exact sequence

$$
0 \rightarrow \Sigma_{\alpha, 1} \rightarrow \operatorname{Mix}_{\alpha, 1} \rightarrow \Sigma_{\alpha, 1} \rightarrow 0 .
$$

(3) The functor $\Sigma_{\alpha, 1}$ is uniserial with unique composition series given by the decreasing filtration given by the subfunctors $k_{d} \Sigma_{\alpha, 1}$ of $\Sigma_{\alpha, 1}$ :

$$
\cdots \subset k_{d} \Sigma_{\alpha, 1} \subset \cdots \subset k_{1} \Sigma_{\alpha, 1} \subset k_{0} \Sigma_{\alpha, 1}=\Sigma_{\alpha, 1} .
$$

(a) The head of $\Sigma_{\alpha, 1}$ (i.e. $\left.\Sigma_{\alpha, 1} / k_{1} \Sigma_{\alpha, 1}\right)$ is isomorphic to the functor $\kappa\left(\operatorname{iso}_{(x, \alpha)}\right)$, where $\operatorname{iso}_{(x, \alpha)}$ is a simple object in $\mathcal{F}_{\text {iso }}$.

(b) For $d>0$,

$$
k_{d} \Sigma_{\alpha, 1} / k_{d+1} \Sigma_{\alpha, 1} \simeq L_{\alpha}^{d+1},
$$

where $L_{\alpha}^{d+1}$ is a simple object of the category $\mathcal{F}_{\text {quad }}$ that is neither in the image of $\iota$ nor in the image of $\kappa$. The functor $L_{\alpha}^{d+1}$ is a subfunctor of $\iota\left(\Lambda^{d+1}\right) \otimes \kappa\left(\operatorname{iso}_{(x, \alpha)}\right)$, where $\Lambda^{d+1}$ is the $(d+1)$ st exterior power functor.

2. Filtration of the standard projective functors $P_{V}$ of $\mathcal{F}_{\text {quad }}$. In this section, we define a filtration of the standard projective functors $P_{V}$ of $\mathcal{F}_{\text {quad }}$. This construction gives rise to an essential tool to obtain, in 
Section 3 , the decompositions of the projective objects $P_{H_{0}}$ and $P_{H_{1}}$ of $\mathcal{F}_{\text {quad }}$ into indecomposable direct summands.

After defining this filtration, we will deduce general results about the projective $P_{V}$ of $\mathcal{F}_{\text {quad }}$. In Theorem 2.6 we prove that the rank zero part is a direct summand of $P_{V}$ and we identify this functor. This result allows us to prove that $\iota(\mathcal{F})$ is a thick subcategory of $\mathcal{F}_{\text {quad }}$. We will also show that the top quotient of this filtration is isomorphic to $\kappa\left(\right.$ iso $\left._{V}\right)$, where iso $V$ is an isotropic functor.

2.1. Definition of the filtration. We recall that a morphism in $\mathcal{T}_{q}$ from $V$ to $W$, where $V$ and $W$ are nondegenerate quadratic spaces, is represented by a diagram $V \rightarrow X \leftarrow W$.

Definition 2.1. A morphism $[V \rightarrow X \leftarrow W]$ in $\mathcal{T}_{q}$ has rank $i$ if the pull-back in $\mathcal{E}_{q}^{\text {deg }}$ of the diagram $V \rightarrow X \leftarrow W$ is a quadratic space of dimension $i$.

Notation 2.2. We denote by $\operatorname{Hom}_{\mathcal{T}_{q}}^{(i)}(V, W)$ the subset of $\operatorname{Hom}_{\mathcal{T}_{q}}(V, W)$ of morphisms of rank less than or equal to $i$.

Proposition 2.3. For any object $W$ in $\mathcal{T}_{q}$, the vector subspace

$$
P_{V}^{(i)}(W)=\mathbb{F}_{2}\left[\operatorname{Hom}_{\mathcal{T}_{q}}^{(i)}(V, W)\right]
$$

of $P_{V}(W)$ defines a subfunctor $P_{V}^{(i)}$ of $P_{V}$.

Proof. It is sufficient to verify that for all morphisms $f=[W \rightarrow Y \leftarrow Z]$ of $\mathcal{T}_{q}$ and $g=[V \rightarrow X \leftarrow W]$ in $\operatorname{Hom}_{\mathcal{T}_{q}}^{(i)}(V, W)$, the composition $f \circ g$ has rank less than or equal to $i$. This composition is represented by the commutative diagram:

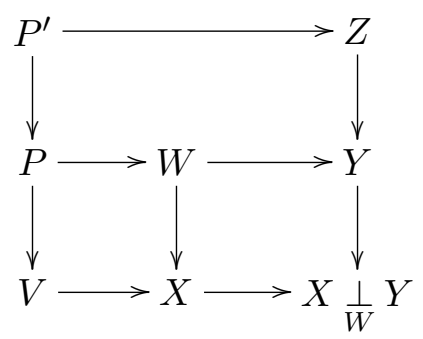

where $P$ and $P^{\prime}$ are the pull-backs and $X \underset{W}{\perp} Y$ is the pseudo push-out defined in [12]. Consequently, $f \circ g=[V \rightarrow X \underset{W}{\perp} Y \leftarrow Z]$. Since $[V \rightarrow X \leftarrow W]$ is an element of $P_{V}^{(i)}(W)$, the dimension of $P$ is less than or equal to $i$.

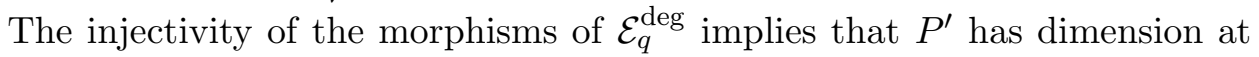
most $i$. 
The following lemma is a straightforward consequence of Definition 2.1.

LEMmA 2.4. There exists a natural equivalence $P_{V}^{(\operatorname{dim}(V))} \simeq P_{V}$.

Proposition 2.5. The functors $P_{V}^{(i)}$ for $i=0, \ldots, \operatorname{dim}(V)$ define an increasing filtration of the functor $P_{V}$.

Proof. The inclusion of vector spaces $P_{V}^{(i)}(W) \subset P_{V}^{(i+1)}(W)$ is clear, for any object $W$ in $\mathcal{T}_{q}$. Consequently, $P_{V}^{(i)}$ is a subfunctor of $P_{V}^{(i+1)}$ by Proposition 2.3.

2.2. Extremities of the filtration. In the previous section, we have obtained, for all objects $V$ of $\mathcal{T}_{q}$, the following filtration of the functor $P_{V}$ :

$$
0 \subset P_{V}^{(0)} \subset P_{V}^{(1)} \subset \cdots \subset P_{V}^{(\operatorname{dim}(V)-1)} \subset P_{V}^{(\operatorname{dim}(V))}=P_{V} .
$$

The aim of this section is to study the two extremities of this filtration, namely, the functor $P_{V}^{(0)}$ and the quotient $P_{V} / P_{V}^{(\operatorname{dim}(V)-1)}$.

2.2.1. The functor $P_{V}^{(0)}$. For any object $V$ in $\mathcal{T}_{q}$, by the Yoneda lemma the functor $P_{\epsilon(V)}^{\mathcal{F}}$ in $\mathcal{F}$ defined by $P_{\epsilon(V)}^{\mathcal{F}}(-)=\mathbb{F}_{2}\left[\operatorname{Hom}_{\mathcal{E}^{f}}(\epsilon(V),-)\right]$ is projective, where $\epsilon: \mathcal{T}_{q} \rightarrow \mathcal{E}^{f}$ is the forgetful functor of $\mathcal{F}_{\text {quad. }}$. The aim of this section is to prove the following theorem:

Theorem 2.6. Let $V$ be an object of $\mathcal{T}_{q}$.

(1) There is a natural equivalence: $P_{V}^{(0)} \simeq \iota\left(P_{\epsilon(V)}^{\mathcal{F}}\right)$, where $\iota: \mathcal{F} \rightarrow \mathcal{F}_{\text {quad }}$ is the functor given in Theorem 1.3.

(2) The functor $P_{V}^{(0)}$ is a direct summand of $P_{V}$.

Before proving this result, we give the following useful characterization of the morphisms of rank zero, which is a straightforward consequence of the definition of the rank of a morphism.

Lemma 2.7. Let $V$ be an object in $\mathcal{T}_{q}$. A morphism $T=\left[V \stackrel{\alpha}{\rightarrow} W \perp W^{\prime}\right.$ $\left.\stackrel{i_{W}}{\longleftarrow} W\right]$ has rank zero if and only if $p_{W^{\prime}} \circ \alpha$ is an injective linear map, where $p_{W^{\prime}}$ is the orthogonal projection from $W \perp W^{\prime}$ to $W^{\prime}$.

For any objects $V$ and $W$ in $\mathcal{T}_{q}$, the forgetful functor $\epsilon: \mathcal{T}_{q} \rightarrow \mathcal{E}^{f}$ gives rise to a map $\operatorname{Hom}_{\mathcal{T}_{q}}(V, W) \rightarrow \operatorname{Hom}_{\mathcal{E}^{f}}(\epsilon(V), \epsilon(W))$. By passage to the vector spaces freely generated by these sets and by functoriality of $\epsilon$, we deduce the existence of a morphism from $P_{V}$ to $\iota\left(P_{\epsilon(V)}^{\mathcal{F}}\right)$. As $P_{V}^{(0)}$ is a subfunctor of $P_{V}$, we obtain a morphism $f$ from $P_{V}^{(0)}$ to $\iota\left(P_{\epsilon(V)}^{\mathcal{F}}\right)$. Consequently, to prove Theorem 2.6(1), it is sufficient to prove the following proposition. 
Proposition 2.8. The map $P_{V}^{(0)}(W) \stackrel{f_{W}}{\longrightarrow} P_{\epsilon(V)}^{\mathcal{F}}(\epsilon(W))$ is an isomorphism for any objects $V$ and $W$ in $\mathcal{T}_{q}$.

The surjectivity of $f_{W}$ relies on the following lemma, which is an improved version of the fullness of the forgetful functor $\epsilon$ given in [12].

Lemma 2.9. Let $\left(V, q_{V}\right)$ and $\left(W, q_{W}\right)$ be two objects of $\mathcal{T}_{q}$ and $f \in$ $\operatorname{Hom}_{\mathcal{E}^{f}}\left(\epsilon\left(V, q_{V}\right), \epsilon\left(W, q_{W}\right)\right)$ a linear map. Then there exists a morphism $T=$ $\left[V \stackrel{\varphi}{\rightarrow} W \perp Y \stackrel{i_{W}}{\longleftarrow} W\right]$ of rank zero such that $\epsilon(T)=f$.

Proof. As the quadratic space $V$ is nondegenerate, it has even dimension, say $2 n$. We prove the result by induction on $n$.

To start the induction, let $\left(V, q_{V}\right)$ be a nondegenerate quadratic space of dimension two, with symplectic basis $\{a, b\}$, and let $f: V \rightarrow W$ be a linear map. The following linear map preserves the quadratic form:

$$
\begin{aligned}
g_{1}: V & \rightarrow W \perp H_{1} \perp H_{0} \perp H_{0} \\
& \simeq W \perp \operatorname{Span}\left(a_{1}, b_{1}\right) \perp \operatorname{Span}\left(a_{0}, b_{0}\right) \perp \operatorname{Span}\left(a_{0}^{\prime}, b_{0}^{\prime}\right), \\
a & \mapsto f(a)+(q(a)+q(f(a))) a_{1}+a_{0}, \\
b & \mapsto f(b)+(q(b)+q(f(b))) a_{1}+(1+B(f(a), f(b))) b_{0}+a_{0}^{\prime} .
\end{aligned}
$$

Consequently, the morphism $T=V \stackrel{g_{1}}{\longrightarrow} W \perp H_{1} \perp H_{0} \hookleftarrow W$ is of rank zero in $\mathcal{T}_{q}$ and $\epsilon(T)=f$.

Let $V_{n}$ be a nondegenerate quadratic space of dimension $2 n$, and let $\left\{a_{1}, b_{1}, \ldots, a_{n}, b_{n}\right\}$ be a symplectic basis of $V_{n}$ and $f_{n}: V_{n} \rightarrow W$ a linear map. By induction, there exists a map

$$
g_{n}: V_{n} \rightarrow W \perp Y, \quad a_{i} \mapsto f_{n}\left(a_{i}\right)+y_{i}, b_{i} \mapsto f_{n}\left(b_{i}\right)+z_{i},
$$

where $y_{i}, z_{i} \in Y$ for all $i \in\{1, \ldots, n\}$. The map $g_{n}$ preserves the quadratic form, and the morphism $T=\left[V_{n} \stackrel{g_{n}}{\longrightarrow} W \perp Y \hookleftarrow W\right]$ is of rank zero and satisfies $\epsilon(T)=f_{n}$.

Let $V_{n+1}$ be a nondegenerate quadratic space of dimension $2(n+1)$, $\left\{a_{1}, b_{1}, \ldots, a_{n}, b_{n}, a_{n+1}, b_{n+1}\right\}$ a symplectic basis of $V_{n+1}$, and $f_{n+1}: V_{n+1} \rightarrow$ $W$ a linear map. To define the map $g_{n+1}$, we will consider the restriction of $f_{n+1}$ to $V_{n}$ and extend the map $g_{n}$ given by the inductive assumption. For that, we need the space

$$
E \simeq W \perp Y \perp H_{0}^{\perp n} \perp H_{0}^{\perp n} \perp H_{1} \perp H_{0} \perp H_{0},
$$

for which we specify the notations for a basis:

$$
\begin{aligned}
E \simeq & W \perp Y \perp\left(\perp_{i=1}^{n} \operatorname{Span}\left(a_{0}^{i}, b_{0}^{i}\right)\right) \perp\left(\perp_{i=1}^{n} \operatorname{Span}\left(A_{0}^{i}, B_{0}^{i}\right)\right) \perp \operatorname{Span}\left(A_{1}, B_{1}\right) \\
& \perp \operatorname{Span}\left(C_{0}, D_{0}\right) \perp \operatorname{Span}\left(E_{0}, F_{0}\right) .
\end{aligned}
$$


The map

$$
\begin{aligned}
g_{n+1}: V \rightarrow & W \perp Y \perp H_{0}^{\perp n} \perp H_{0}^{\perp n} \perp H_{1} \perp H_{0} \perp H_{0}, \\
a_{i} \mapsto & f_{n+1}\left(a_{i}\right)+y_{i}+a_{0}^{i}, \\
b_{i} \mapsto & f_{n+1}\left(b_{i}\right)+z_{i}+A_{0}^{i} \quad \text { for } i \in\{1, \ldots, n\}, \\
a_{n+1} \mapsto & f_{n+1}\left(a_{n+1}\right)+\left(q\left(a_{n+1}\right)+q\left(f_{n+1}\left(a_{n+1}\right)\right)\right) A_{1}+C_{0} \\
& +\sum_{i=1}^{n} B\left(f_{n+1}\left(a_{i}\right), f_{n+1}\left(a_{n+1}\right)\right) b_{0}^{i} \\
& +\sum_{i=1}^{n} B\left(f_{n+1}\left(b_{i}\right), f_{n+1}\left(a_{n+1}\right)\right) B_{0}^{i}, \\
b_{n+1} \mapsto & f_{n+1}\left(b_{n+1}\right)+\left(q\left(b_{n+1}\right)+q\left(f_{n+1}\left(b_{n+1}\right)\right)\right) A_{1} \\
& +\left(1+B\left(f_{n+1}\left(a_{n+1}\right), f_{n+1}\left(b_{n+1}\right)\right)\right) D_{0} \\
& +\sum_{i=1}^{n} B\left(f_{n+1}\left(a_{i}\right), f_{n+1}\left(b_{n+1}\right)\right) b_{0}^{i} \\
& +\sum_{i=1}^{n} B\left(f_{n+1}\left(b_{i}\right), f_{n+1}\left(b_{n+1}\right)\right) B_{0}^{i}+E_{0},
\end{aligned}
$$

preserves the quadratic form. Furthermore, the morphism

$$
T=\left[V_{n+1} \stackrel{g_{n+1}}{\longrightarrow} W \perp Y \perp H_{0}^{\perp n} \perp H_{0}^{\perp n} \perp H_{1} \perp H_{0} \perp H_{0} \hookleftarrow W\right]
$$

is of rank zero and satisfies $\epsilon(T)=f_{n+1}$, which completes the inductive step.

The proof of the injectivity of $f_{W}$ relies on the following result, which can be regarded as Witt's theorem for degenerate quadratic forms.

Theorem 2.10 ([2, $\S 4$, Theorem 1$])$. Let $V$ be a nondegenerate quadratic space, $D$ and $D^{\prime}$ quadratic subspaces (possibly degenerate) of $V$, and $\underline{f}$ : $D \rightarrow D^{\prime}$ an isometry. Then there exists an isometry $f: V \rightarrow V$ extending $\underline{f}$.

Proof of the injectivity of $f_{W}$. The natural map $f$ is induced by the natural map

$$
\operatorname{Hom}_{\mathcal{T}_{q}}^{(0)}(V,-) \rightarrow \operatorname{Hom}_{\mathcal{E}^{f}}(\epsilon(V), \epsilon(-))
$$

by passage to the vector spaces freely generated by these sets. So, $f$ is injective if and only if this natural map is injective. Consequently, it is sufficient to verify that, for two generators $T=\left[V \stackrel{\alpha}{\rightarrow} W \perp W^{\prime} \stackrel{i_{W}}{\longleftarrow} W\right]$ and $T^{\prime}=\left[V \stackrel{\alpha^{\prime}}{\longrightarrow} W \perp W^{\prime \prime} \stackrel{i_{W}^{\prime}}{\longleftarrow} W\right]$ of $P_{V}^{(0)}(W)$ such that

$$
p_{W} \circ \mathcal{O}(\alpha)=p_{W}^{\prime} \circ \mathcal{O}\left(\alpha^{\prime}\right) \text {, }
$$

we have $T=T^{\prime}$. 
Let $\left\{a_{1}, b_{1}, \ldots, a_{n}, b_{n}\right\}$ be a symplectic basis of $V$. We deduce from (2.10.1) that, for all $i \in\{1, \ldots, n\}$,

$$
\begin{aligned}
\alpha\left(a_{i}\right) & =w_{i}+w_{i}^{\prime}, & \alpha\left(b_{i}\right) & =x_{i}+x_{i}^{\prime}, \\
\alpha^{\prime}\left(a_{i}\right) & =w_{i}+w_{i}^{\prime \prime}, & \alpha^{\prime}\left(b_{i}\right) & =x_{i}+x_{i}^{\prime \prime},
\end{aligned}
$$

where, for all $i, w_{i}$ and $x_{i}$ are in $W, w_{i}^{\prime}$ and $x_{i}^{\prime}$ are in $W^{\prime}$, and $x_{i}^{\prime \prime}$ and $w_{i}^{\prime \prime}$ are in $W^{\prime \prime}$. By Lemma 2.7, since the relevant morphisms are of rank zero, $\left\{w_{1}^{\prime}, x_{1}^{\prime}, \ldots, w_{n}^{\prime}, x_{n}^{\prime}\right\}$ and $\left\{w_{1}^{\prime \prime}, x_{1}^{\prime \prime}, \ldots, w_{n}^{\prime \prime}, x_{n}^{\prime \prime}\right\}$ are two linearly independent families of vectors.

We will denote by $\underline{W}^{\prime}=\operatorname{Span}\left(w_{1}^{\prime}, x_{1}^{\prime}, \ldots, w_{n}^{\prime}, x_{n}^{\prime}\right)$ (respectively $\underline{W}^{\prime \prime}=$ $\left.\operatorname{Span}\left(w_{1}^{\prime \prime}, x_{1}^{\prime \prime}, \ldots, w_{n}^{\prime \prime}, x_{n}^{\prime \prime}\right)\right)$ the quadratic subspace (possibly degenerate) of $W^{\prime}$ (respectively $W^{\prime \prime}$ ) and we define the linear map $\underline{f}: \underline{W}^{\prime} \rightarrow \underline{W}^{\prime \prime}$ by $\underline{f}\left(w_{i}^{\prime}\right)=w_{i}^{\prime \prime}$ and $\underline{f}\left(x_{i}^{\prime}\right)=x_{i}^{\prime \prime}$ for all $i \in\{1, \ldots, n\}$.

Since $\alpha$ and $\alpha^{\prime}$ preserve the quadratic forms, we deduce from (2.10.2) and (2.10.3) that $\underline{f}$ preserves the quadratic form. Hence, we can apply Theorem 2.10 to the nondegenerate space $W^{\prime} \perp W^{\prime \prime}$, which gives a morphism $f: W^{\prime} \perp W^{\prime \prime} \rightarrow W^{\prime} \perp W^{\prime \prime}$ in $\mathcal{E}_{q}$, extending $\underline{f}$. This yields the commutativity of the diagram

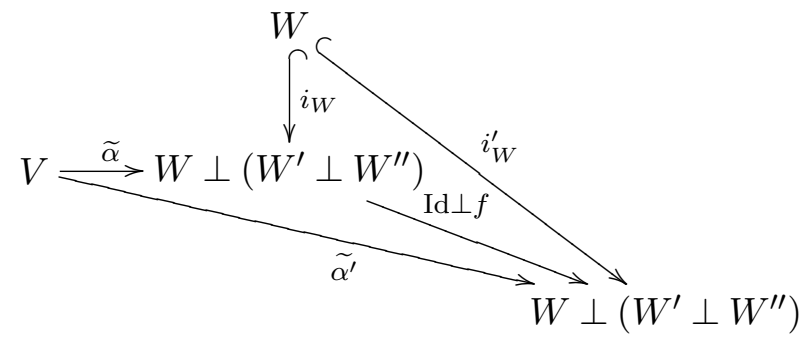

where $\widetilde{\alpha}=i_{W \perp W^{\prime}} \circ \alpha$ and $\widetilde{\alpha^{\prime}}=i_{W \perp W^{\prime \prime}} \circ \alpha^{\prime}$. Consequently, $T=T^{\prime}$ since, by inclusion, we have

$$
\begin{aligned}
T & =\left[V \stackrel{\alpha}{\rightarrow} W \perp W^{\prime} \stackrel{i_{W}}{\longleftarrow} W\right]=\left[V \stackrel{\widetilde{\alpha}}{\rightarrow} W \perp W^{\prime} \perp W^{\prime \prime} \stackrel{i_{W}}{\longleftarrow} W\right], \\
T^{\prime} & =\left[V \stackrel{\alpha^{\prime}}{\longrightarrow} W \perp W^{\prime \prime} \stackrel{i_{W}^{\prime}}{\longleftarrow} W\right]=\left[V \stackrel{\widetilde{\alpha}^{\prime}}{\longrightarrow} W \perp W^{\prime} \perp W^{\prime \prime} \stackrel{i_{W}^{\prime}}{\longleftarrow} W\right] .
\end{aligned}
$$

Notation 2.11. For any two objects $V$ and $W$ of $\mathcal{E}_{q}$, and for any $f \in \operatorname{Hom}_{\mathcal{E} f}(\epsilon(V), \epsilon(W))$, we denote by $t_{f}$ the morphism in $\operatorname{Hom}_{\mathcal{T}_{q}}^{(0)}(V, W)$ corresponding to $f$ and by $\left[t_{f}\right]$ the canonical generator of $P_{V}^{(0)}(W)$ obtained from $t_{f}$. To simplify notation, we denote $t_{\mathrm{Id}_{V}} \in \operatorname{Hom}_{\mathcal{T}_{q}}^{(0)}(V, V)$ by $e_{V}$.

Theorem 2.6(1) yields the following corollary.

Corollary 2.12. For any objects $V, W$ and $X$ of $\mathcal{E}_{q}$, and morphisms $f: \epsilon(W) \rightarrow \epsilon(X)$ and $g: \epsilon(V) \rightarrow \epsilon(W)$ of $\mathcal{E}^{f}$, we have

$$
t_{f} \circ t_{g}=t_{f \circ g}
$$


where $t_{f}, t_{g}$ and $t_{f \circ g}$ are respectively the morphisms in $\operatorname{Hom}_{\mathcal{T}_{q}}^{(0)}(W, X)$, $\operatorname{Hom}_{\mathcal{T}_{q}}^{(0)}(V, W)$ and $\operatorname{Hom}_{\mathcal{T}_{q}}^{(0)}(V, X)$ associated to the linear maps $f, g$ and $f \circ g$.

We can apply this result to the idempotents of the ring of endomorphisms $\operatorname{End}\left(P_{V}\right)$, to obtain the following proposition.

Proposition 2.13. The canonical generator $\left[e_{V}\right]$ of $P_{V}^{(0)}$ is an idempotent of the ring of endomorphisms $\operatorname{End}\left(P_{V}\right)$ such that $P_{V} \cdot\left[e_{V}\right] \simeq P_{V}^{(0)}$. Consequently, $P_{V}^{(0)}$ is a direct summand of $P_{V}$.

Proof. The canonical generator $\left[e_{V}\right]$ is an idempotent of $\operatorname{End}\left(P_{V}\right)$ by Corollary 2.12. By definition of the rank filtration, $P_{V} \cdot\left[e_{V}\right] \subset P_{V}^{(0)}$ and, for a canonical generator $\left[t_{f}\right]$ of $P_{V}^{(0)}$, we have $\left[t_{f}\right]=\left[t_{f}\right] \cdot\left[e_{V}\right]$.

Since the idempotents $\left[e_{V}\right]$ and $1-\left[e_{V}\right]$ of $\operatorname{End}\left(P_{V}\right)$ are orthogonal and satisfy $\left[e_{V}\right]+\left(1-\left[e_{V}\right]\right)=1$, we obtain the following decomposition of $P_{V}$ into direct summands: $P_{V}=P_{V} \cdot\left[e_{V}\right] \oplus P_{V} \cdot\left(1-\left[e_{V}\right]\right)$. Hence $P_{V}^{(0)}$ is a direct summand of $P_{V}$.

The idempotent $\left[e_{V}\right]$ plays a central rôle in the proof of the thickness of the subcategory $\iota(\mathcal{F})$ in $\mathcal{F}_{\text {quad }}$, which is the subject of the following section. For that, the following result is necessary.

Lemma 2.14. Let $V$ and $W$ be objects of $\mathcal{T}_{q}$. Then the functor $\iota$ induces an isomorphism

$$
\operatorname{Hom}_{\mathcal{F}}\left(P_{\epsilon(V)}^{\mathcal{F}}, P_{\epsilon(W)}^{\mathcal{F}}\right) \stackrel{\simeq}{\longrightarrow} \operatorname{Hom}_{\mathcal{F}_{\text {quad }}}\left(P_{V}^{(0)}, P_{W}^{(0)}\right),
$$

where $\epsilon: \mathcal{T}_{q} \rightarrow \mathcal{E}$ is the forgetful functor.

Proof. By Proposition 2.13 and Theorem 2.6 we have

$$
\begin{aligned}
\operatorname{Hom}_{\mathcal{F}_{\text {quad }}}\left(P_{V}^{(0)}, P_{W}^{(0)}\right) & \simeq P_{W}^{(0)}\left(e_{V}\right) P_{W}^{(0)}(V) \simeq P_{W}^{(0)}(V) \\
& \simeq \iota\left(P_{\epsilon(W)}^{\mathcal{F}}\right)(V) \simeq P_{\epsilon(W)}^{\mathcal{F}}(\epsilon(V)) \simeq \operatorname{Hom}_{\mathcal{F}}\left(P_{\epsilon(V)}^{\mathcal{F}}, P_{\epsilon(W)}^{\mathcal{F}}\right)
\end{aligned}
$$

To conclude this section, we give the following property of $e_{V}$ which will be useful in Section 4 concerning the polynomial functors in $\mathcal{F}_{\text {quad }}$.

LEMMA 2.15. For any two objects $V$ and $W$ of $\mathcal{T}_{q}$, we have $e_{V \perp W}=$ $e_{V} \perp e_{W}$, where $\perp: \mathcal{T}_{q} \times \mathcal{T}_{q} \rightarrow \mathcal{T}_{q}$ is the functor induced by the orthogonal sum.

Proof. This is a straightforward consequence of Proposition 2.8.

2.2.2. The category $\iota(\mathcal{F})$ is a thick subcategory of $\mathcal{F}_{\text {quad }}$. Recall that a subcategory $\mathcal{C}$ of an abelian category $\mathcal{A}$ is thick if $\mathcal{C}$ is a full subcategory which contains all subobjects and quotients of its objects and is closed under formation of extensions. The aim of this section is to prove the following result. 
TheOREM 2.16. The category $\iota(\mathcal{F})$ is a thick subcategory of $\mathcal{F}_{\text {quad }}$, where $\iota: \mathcal{F} \rightarrow \mathcal{F}_{\text {quad }}$ is the functor defined in Theorem 1.3.

To prove this theorem, we need the following general result about the precomposition functor which is proved in the Appendix of [12].

Proposition 2.17. Let $\mathcal{C}$ and $\mathcal{D}$ be two small categories, $\mathcal{A}$ be an abelian category, $F: \mathcal{C} \rightarrow \mathcal{D}$ be a functor and $-\circ F: \operatorname{Func}(\mathcal{D}, \mathcal{A}) \rightarrow \operatorname{Func}(\mathcal{C}, \mathcal{A})$ be the precomposition functor, where $\operatorname{Func}(\mathcal{C}, \mathcal{A})$ is the category of functors from $\mathcal{C}$ to $\mathcal{A}$. If $F$ is full and essentially surjective, then any subobject (respectively quotient) of an object in the image of the precomposition functor is isomorphic to an object in that image.

Proof of Theorem 2.16. • The subcategory $\iota(\mathcal{F})$ of $\mathcal{F}_{\text {quad }}$ is full by Theorem 1.3.

- Let $F^{\mathcal{F}}$ be an object in $\mathcal{F}$, and $G$ a subobject of $\iota\left(F^{\mathcal{F}}\right)$. Let $\mathcal{F}^{\prime}$ be

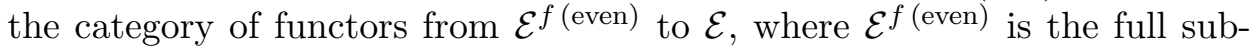
category of $\mathcal{E}^{f}$ having as objects the $\mathbb{F}_{2}$-vector spaces of even dimension. The categories $\mathcal{F}$ and $\mathcal{F}^{\prime}$ are equivalent [12]. The functor $\epsilon: \mathcal{E}_{q} \rightarrow \mathcal{E}^{f}$

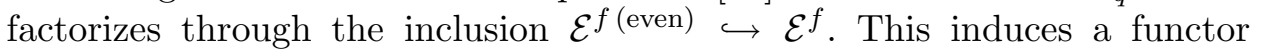
$\epsilon^{\prime}: \mathcal{E}_{q} \rightarrow \mathcal{E}^{f(\text { even })}$ which is full and essentially surjective. Consequently, we can use Proposition 2.17 to obtain $G \simeq \iota\left(G^{\mathcal{F}}\right)$. Similarly, we obtain the result for the quotient.

- Let $G^{\mathcal{F}}$ and $H^{\mathcal{F}}$ be objects of $\mathcal{F}$, and set $G=\iota\left(G^{\mathcal{F}}\right)$ and $H=\iota\left(H^{\mathcal{F}}\right)$. For any short exact sequence $0 \rightarrow G \rightarrow F \rightarrow H \rightarrow 0$, we have to prove that there exists a functor $F^{\mathcal{F}}$ in $\mathcal{F}$ such that $F=\iota\left(F^{\mathcal{F}}\right)$.

Let $P_{1} \rightarrow P_{0} \rightarrow G^{\mathcal{F}} \rightarrow 0$ and $Q_{1} \rightarrow Q_{0} \rightarrow H^{\mathcal{F}} \rightarrow 0$ be projective presentations of $G^{\mathcal{F}}$ and $H^{\mathcal{F}}$ in $\mathcal{F}$. We have the following commutative diagram:

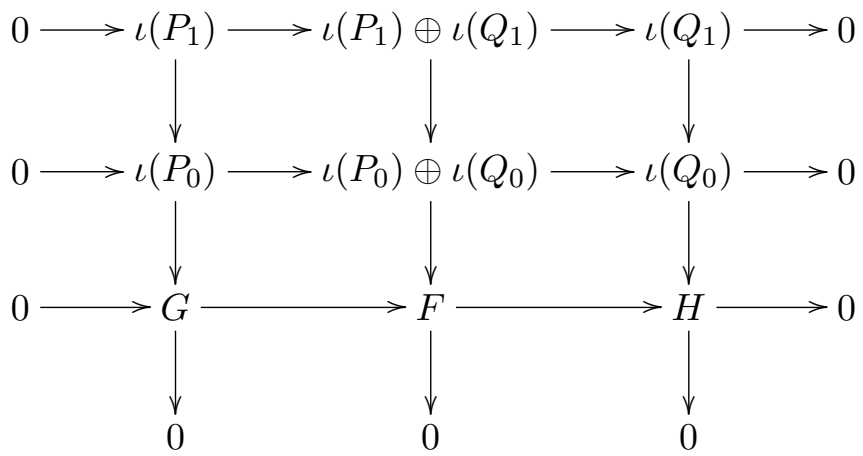

where the columns are projective resolutions in $\mathcal{F}_{\text {quad }}$, by the horseshoe lemma. By Lemma 2.14, the morphism $\iota\left(P_{1}\right) \oplus \iota\left(Q_{1}\right) \rightarrow \iota\left(P_{0}\right) \oplus \iota\left(Q_{0}\right)$ is induced by a morphism of $\mathcal{F}$ denoted by $f$. Consequently, $F \cong \iota(\operatorname{Coker}(f))$ $\in \iota(\mathcal{F})$. 
By Theorem 2.16, we deduce from Lemma 2.14 the following characterization of the simple functors of $\mathcal{F}$ in $\mathcal{F}_{\text {quad }}$ which will be used in Section 4 concerning the polynomial functors in $\mathcal{F}_{\text {quad }}$.

LEMMA 2.18.

(1) Let $F$ be a functor in $\mathcal{F}_{\text {quad. }}$. Then $F$ is in the image of the functor $\iota: \mathcal{F} \rightarrow \mathcal{F}_{\text {quad }}$ if and only if, for all objects $V$ in $\mathcal{T}_{q}$,

$$
F\left(e_{V}\right) F(V)=F(V) .
$$

(2) Let $S$ be a simple object in $\mathcal{F}_{\text {quad. }}$. Then $S$ is in the image of $\iota$ if and only if there exists an object $W$ in $\mathcal{T}_{q}$ such that

$$
S\left(e_{W}\right) S(W) \neq 0 .
$$

Proof. (1) The forward implication is a consequence of the following fact: for a functor $F$ in the image of $\iota, \operatorname{Hom}_{\mathcal{F}_{\text {quad }}}\left(P_{V}^{(0)}, F\right)=F\left(e_{V}\right) F(V)=F(V)$. The reverse implication relies on the fact that the condition $F\left(e_{V}\right) F(V)$ $=F(V)$ implies that $F$ is a quotient of a sum of projective objects of the form $P_{V}^{(0)}$. Since the category $\iota(\mathcal{F})$ is thick in $\mathcal{F}_{\text {quad }}$ by Theorem 2.16, we obtain the result.

(2) Observe that, if $S\left(e_{W}\right) S(W) \neq 0$, we have $\operatorname{Hom}_{\mathcal{F}_{\text {quad }}}\left(P_{W}^{(0)}, S\right) \neq 0$, thus $S$ is a quotient of $P_{W}^{(0)}$ by simplicity of $S$. Lemma 2.14 implies that there exists a one-to-one correspondence between the indecomposable factors of $P_{V}^{(0)}$ and those of $P_{\epsilon(V)}^{\mathcal{F}}$. We deduce that the simple quotients of $P_{V}^{(0)}$ arise from $\mathcal{F}$. Consequently, $S$ is in the image of the functor $\iota$.

2.2.3. The quotient $P_{V} / P_{V}^{(\operatorname{dim}(V)-1)}$. The aim of this section is to prove the following result:

Proposition 2.19. Let $V$ be an object in $\mathcal{T}_{q}$. We have a natural equivalence

$$
P_{V} / P_{V}^{(\operatorname{dim}(V)-1)} \simeq \kappa\left(\text { iso }_{V}\right),
$$

where iso $V$ is an isotropic functor and $\kappa: \mathcal{F}_{\text {iso }} \rightarrow \mathcal{F}_{\text {quad }}$ is the functor given in Theorem 1.4.

To prove this proposition, we need the following notation and result:

Notation 2.20. Denote by $\sigma_{f}$ the natural map $P_{V} \stackrel{\sigma_{f}}{\longrightarrow} \kappa($ iso $V)$ which corresponds to the canonical generator $[V \stackrel{\mathrm{Id}}{\longleftarrow} V \stackrel{f}{\rightarrow} V]$ of iso $_{V}(V)$ via the equivalence $\operatorname{Hom}\left(P_{V}, \kappa\left(\right.\right.$ iso $\left.\left._{V}\right)\right) \simeq \operatorname{iso}_{V}(V) \simeq \mathbb{F}_{2}[O(V)]$ given by the Yoneda lemma.

Lemma 2.21. The functor $\kappa\left(\right.$ iso $\left._{V}\right)$ of $\mathcal{F}_{\text {quad }}$ is a quotient of the functor $P_{V}=\mathbb{F}_{2}\left[\operatorname{Hom}_{\mathcal{T}_{q}}(V,-)\right]$. 
Proof. The natural map $P_{V} \stackrel{\sigma_{f}}{\longrightarrow} \kappa\left(\right.$ iso $\left._{V}\right)$ is surjective: a preimage of the canonical generator $[V \stackrel{\text { Id }}{\longleftarrow} V \stackrel{g}{\rightarrow} W]$ of $\kappa\left(\right.$ iso $\left._{V}\right)(W)$ under $\left(\sigma_{f}\right)_{W}$ is the morphism $\left[V \stackrel{g \circ f^{-1}}{\longrightarrow} W \stackrel{\mathrm{Id}}{\longleftarrow} W\right]$.

A formal consequence of the previous lemma is given in the following result.

Lemma 2.22. The functor $\kappa\left(\right.$ iso $\left._{V}\right)$ in $\mathcal{F}_{\text {quad }}$ is a quotient of the functor $P_{V} / P_{V}^{(\operatorname{dim}(V)-1)}$.

Proof. By definition of the filtration and by the previous lemma, we have the diagram

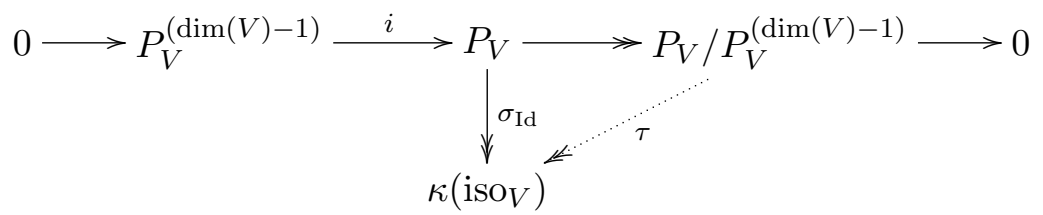

where $i$ is the canonical inclusion. By definition of $\sigma_{\mathrm{Id}}$, we have $\sigma_{\mathrm{Id}} \circ i=0$, which yields a surjection $\tau: P_{V} / P_{V}^{(\operatorname{dim}(V)-1)} \rightarrow \kappa\left(\right.$ iso $\left._{V}\right)$.

We will prove below that this natural map is an isomorphism. It is sufficient to prove the following result.

Proposition 2.23. For any two objects $V$ and $W$ of $\mathcal{T}_{q}$, we have

$$
\left(P_{V} / P_{V}^{(\operatorname{dim}(V)-1)}\right)(W) \simeq \kappa\left(\operatorname{iso}_{V}\right)(W) .
$$

The proof of this proposition relies on the following lemma.

LEMma 2.24. For a nonzero canonical generator of $\left(P_{V} / P_{V}^{(\operatorname{dim}(V)-1)}\right)(W)$ represented by the morphism $T=\left[V \stackrel{g}{\rightarrow} W \perp W^{\prime} \stackrel{i_{W}}{\longleftarrow} W\right]$ of $\mathcal{T}_{q}$, we have $g(V) \subset W$ and $T=[V \stackrel{f}{\longrightarrow} W \stackrel{\text { Id }}{\longleftarrow} W]$, where $g=i_{W} \circ f$.

Proof. By definition of the filtration, for any two objects $V$ and $W$ of $\mathcal{T}_{q}$, the vector space $\left(P_{V} / P_{V}^{(\operatorname{dim}(V)-1)}\right)(W)$ is generated by $\operatorname{Hom}_{\mathcal{T}_{q}}^{[\operatorname{dim}(V)]}(V, W)$, the set of morphisms from $V$ to $W$ whose pull-back $D$ in $\mathcal{E}_{q}^{\text {deg }}$ is a quadratic space such that $\operatorname{dim}(D)=\operatorname{dim}(V)$. We deduce from the existence of a monomorphism from $D$ to $V$ and from the equality of the dimensions that $D$ and $V$ are isometric. Consequently, for the morphism $T$ of the statement, we have, by definition of the pull-back, $g(V) \subset W$. Thus, $T=[V \stackrel{f}{\rightarrow} W \stackrel{\text { Id }}{\longleftarrow} W]$, where $g=i_{W} \circ f$, by the equivalence relation between morphisms of $\mathcal{T}_{q}$, described in Definition 1.1. 
Proof of Proposition 2.23. The natural map $\tau$ obtained in the proof of Lemma 2.22 defines, for any object $W$ in $\mathcal{T}_{q}$, the linear map

$$
\begin{aligned}
\tau_{W}:\left(P_{V} / P_{V}^{(\operatorname{dim}(V)-1)}\right)(W) & \rightarrow \kappa\left(\text { iso }_{V}\right)(W), \\
T=[V \stackrel{f}{\rightarrow} W \stackrel{\text { Id }}{\longleftarrow} W] & \mapsto V \stackrel{\text { Id }}{\longleftarrow} V \stackrel{f}{\rightarrow} W],
\end{aligned}
$$

which is clearly an isomorphism.

3. Decomposition of the standard projective functors $P_{H_{0}}$ and $P_{H_{1}}$. On abelian categories, decompositions of a functor $F$ into direct summands in $\mathcal{F}_{\text {quad }}$ correspond to decompositions of 1 into orthogonal idempotents in the ring $\operatorname{End}_{\mathcal{F}_{\text {quad }}}(F)$ (see for example [3]). One of the difficulties of the category $\mathcal{F}_{\text {quad }}$ lies in the fact that the rings of endomorphisms of projectives $P_{V}$ and their representations are not well understood. The decompositions of projectives $P_{V}$, obtained in work in preparation, using a refinement of the rank filtration, will allow us to understand the structure of those rings better.

In this section, we obtain the decompositions into indecomposable factors of the projective objects $P_{H_{0}}$ and $P_{H_{1}}$ by an explicit study of the filtration defined in Section 2. The section concludes with several consequences of these decompositions. In particular, we give a classification of the "small" simple functors of $\mathcal{F}_{\text {quad }}$, which is an essential ingredient in the following section about the polynomial functors in $\mathcal{F}_{\text {quad }}$.

3.1. Decomposition of $P_{H_{0}}$. To obtain the decomposition of the functor $P_{H_{0}}$ into indecomposable factors, we give an explicit description of the subquotients of the filtration; then we prove that the filtration splits for this functor and we identify the factors of this decomposition.

3.1.1. Explicit description of the subquotients of the filtration. The aim of this section is to give bases of the vector spaces $P_{H_{0}}^{(0)}(V), P_{H_{0}}^{(1)} / P_{H_{0}}^{(0)}(V)$ and $P_{H_{0}} / P_{H_{0}}^{(1)}(V)$ for any given object $V$ in $\mathcal{T}_{q}$.

We deduce from Theorem 2.6 and Notation 2.11 the following result.

Lemma 3.1. A basis of $P_{H_{0}}^{(0)}(V)$ is given by the set

$$
\mathcal{B}_{H_{0}}^{(0)}=\left\{\left[t_{f}\right] \mid f \in \operatorname{Hom}_{\mathcal{E}^{f}}\left(\mathbb{F}_{2}{ }^{\oplus 2}, \epsilon(V)\right)\right\} .
$$

By definition of the filtration, a canonical generator of $P_{H_{0}}^{(1)} / P_{H_{0}}^{(0)}(V)$, represented by the morphism $T=\left[H_{0} \stackrel{f}{\rightarrow} V \perp L \stackrel{i_{V}}{\longleftarrow} V\right]$ of $\mathcal{T}_{q}$, has the property that

$$
I=f\left(H_{0}\right) \cap i(V)
$$

is a quadratic space of dimension one. 
LEMmA 3.2. Let $T=\left[H_{0} \stackrel{f}{\rightarrow} V \perp L \stackrel{i_{V}}{\longleftarrow} V\right]$ be a morphism of $\mathcal{T}_{q}$ which represents a canonical generator of $P_{H_{0}}^{(1)} / P_{H_{0}}^{(0)}(V)$, and $\left\{a_{0}, b_{0}\right\}$ be a symplectic basis of $H_{0}$. Then the map $f$ in $T$ has one of the following three forms.

(1) If $I=\left(f\left(a_{0}\right), 0\right)$, then $f$ is defined by

$$
f\left(a_{0}\right)=v \quad \text { and } \quad f\left(b_{0}\right)=w+l
$$

for some $v, w \in V$ satisfying $q(v)=0$ and $B(v, w)=1$ and some $l \in L \backslash\{0\}$.

(2) If $I=\left(f\left(b_{0}\right), 0\right)$, then $f$ is defined by

$$
f\left(a_{0}\right)=v+l \quad \text { and } \quad f\left(b_{0}\right)=w
$$

for some $v, w \in V$ with $q(w)=0$ and $B(v, w)=1$, and some $l \in L \backslash\{0\}$.

(3) If $I=\left(f\left(a_{0}+b_{0}\right), 1\right)$, then $f$ is defined by

$$
f\left(a_{0}\right)=v+l \quad \text { and } \quad f\left(b_{0}\right)=w+l
$$

for $v, w \in V$ with $q(v+w)=1$ and $B(v, w)=1$, and some $l \in L \backslash\{0\}$.

Proof. The quadratic space $H_{0}$ has three subspaces of dimension one: $\operatorname{Span}\left(a_{0}\right)$ and $\operatorname{Span}\left(b_{0}\right)$ isometric to $(x, 0)$, and $\operatorname{Span}\left(a_{0}+b_{0}\right)$ isometric to $(x, 1)$. These three subspaces give rise to the respective maps $f$ defined in the statement.

Notation 3.3. The morphisms $\left[H_{0} \stackrel{f}{\rightarrow} V \perp L \stackrel{i_{V}}{\longleftarrow} V\right]$, where $f$ is as in item (1) (respectively (2) and (3)) of the previous lemma, will be referred to as type $A$ (respectively $B$ and $C$ ) morphisms.

Proposition 3.4. For morphisms $T=\left[H_{0} \stackrel{f}{\rightarrow} V \perp L \stackrel{i_{V}}{\longleftarrow} V\right]$ and $T^{\prime}=$ $\left[H_{0} \stackrel{f^{\prime}}{\longrightarrow} V \perp L^{\prime} \stackrel{i_{V}^{\prime}}{\longleftarrow} V\right]$ of $\mathcal{T}_{q}$ which represent canonical generators of $P_{H_{0}}^{(1)} / P_{H_{0}}^{(0)}(V)$, the following properties are equivalent.

(1) $T$ and $T^{\prime}$ have the same type and satisfy $p_{V} \circ f=p_{V}^{\prime} \circ f^{\prime}$.

(2) $T=T^{\prime}$.

The proof of $(2) \Rightarrow(1)$ relies on the following technical lemma.

LEMmA 3.5. Let $T=[V \stackrel{g}{\rightarrow} X \stackrel{h}{\longleftarrow} W]$ and $T^{\prime}=\left[V \stackrel{g^{\prime}}{\longrightarrow} X^{\prime} \stackrel{h^{\prime}}{\longleftarrow} W^{\prime}\right]$ be morphisms in $\operatorname{Hom}_{\mathcal{T}_{q}}(V, W)$. If $T=T^{\prime}$, then $g(V)+h(W) \simeq g^{\prime}(V)+h^{\prime}(W)$ in $\mathcal{E}_{q}^{\mathrm{deg}}$.

Proof. By definition of the equivalence relation in Definition 1.1, it is sufficient to prove that, for two morphisms $T$ and $T^{\prime}$ such that $T \mathcal{R} T^{\prime}$, we have $g(V)+h(W) \simeq g^{\prime}(V)+h^{\prime}(W)$. 
By definition, $g(V)+h(W)$ is the smallest, possibly degenerate, quadratic space such that we have a commutative diagram in $\mathcal{E}_{q}^{\text {deg }}$ of the form

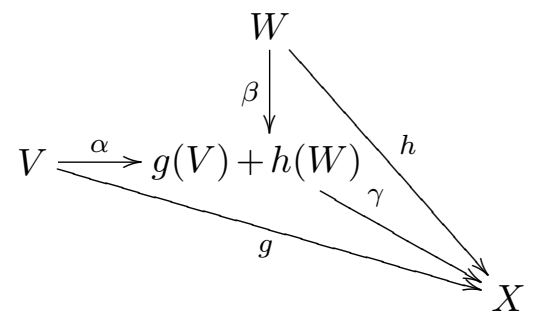

Similarly, $g^{\prime}(V)+h^{\prime}(W)$ is the smallest quadratic space such that we have an analogous commutative diagram. By definition of the relation $\mathcal{R}$, there exists a morphism $\delta$ in $\mathcal{E}_{q}$ such that the following diagram is commutative:

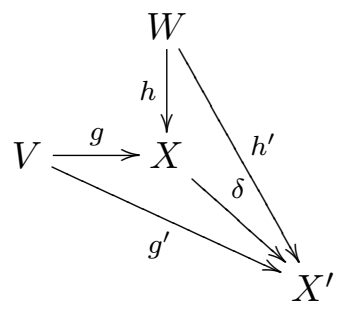

By the consideration of the following commutative diagram in $\mathcal{E}_{q}^{\text {deg. }}$

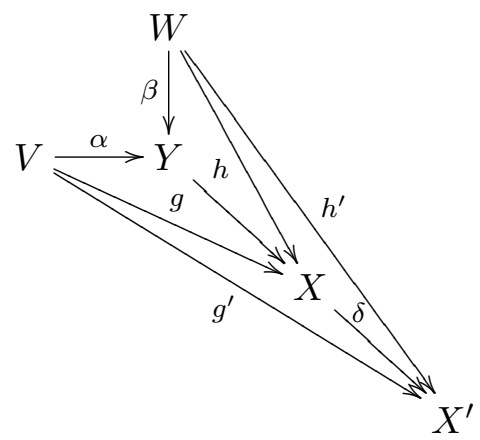

where $Y=g(V)+h(W)$, we deduce from the minimality of $g^{\prime}(V)+h^{\prime}(W)$ the existence of a morphism in $\mathcal{E}_{q}^{\text {deg }}$ from $g^{\prime}(V)+h^{\prime}(W)$ to $g(V)+h(W)$ such that the corresponding diagram is commutative. Then, by minimality of $g(V)+h(W)$ for $T$, we obtain $g(V)+h(W) \simeq g^{\prime}(V)+h^{\prime}(W)$.

Proof of Proposition 3.4. Suppose that the morphisms $T$ and $T^{\prime}$ in $\operatorname{Hom}_{\mathcal{T}_{q}}\left(H_{0}, V\right)$ are of type $A$ and $p_{V} \circ f=p_{V}^{\prime} \circ f^{\prime}$. Then

$$
f\left(a_{0}\right)=v, f\left(b_{0}\right)=w+l \text { and } f^{\prime}\left(a_{0}\right)=v, f^{\prime}\left(b_{0}\right)=w+l^{\prime}
$$

for some $v, w \in V$ and $l \in L \backslash\{0\}, l^{\prime} \in L^{\prime} \backslash\{0\}$. 
Since the maps $f$ and $f^{\prime}$ preserve the quadratic forms, we have $q\left(b_{0}\right)=$ $q(w)+q(l)=q(w)+q\left(l^{\prime}\right)$. Hence $q(l)=q\left(l^{\prime}\right)$, and thus the map $\operatorname{Span}(l) \stackrel{\underline{\alpha}}{\rightarrow}$ $\operatorname{Span}\left(l^{\prime}\right)$ such that $\underline{\alpha}(l)=l^{\prime}$, preserves the quadratic form. Consequently, Theorem 2.10 yields a map $\alpha: L \perp L^{\prime} \rightarrow L \perp L^{\prime}$ extending $\underline{\alpha}$.

This implies the commutativity of the diagram

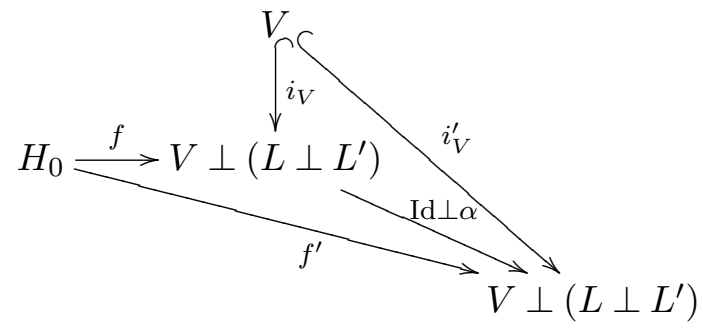

Since $T=\left[H_{0} \stackrel{f}{\rightarrow} V \perp L \perp L^{\prime} \stackrel{i_{V}}{\longleftarrow} V\right]$ and $T^{\prime}=\left[H_{0} \stackrel{f^{\prime}}{\longrightarrow} V \perp L \perp L^{\prime} \stackrel{i_{V}^{\prime}}{\longleftarrow} V\right]$, by inclusion, we deduce from the previous diagram that $T=T^{\prime}$.

We reason in the same way for morphisms of type $B$ and $C$.

Conversely, if $T=T^{\prime}$, then $f\left(H_{0}\right)+i_{V}(V) \simeq f^{\prime}\left(H_{0}\right)+i_{V}^{\prime}(V)$ by Lemma 3.5. Consequently, Theorem 2.10 yields an isometry $\beta: V \perp L \perp L^{\prime} \rightarrow$ $V \perp L \perp L^{\prime}$ making the following diagram commutative:

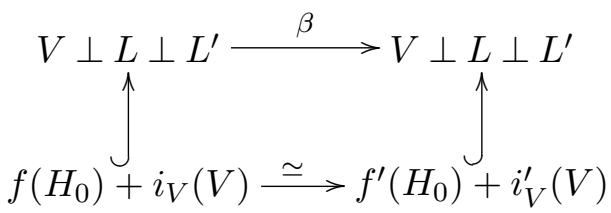

This yields the commutativity of the diagram

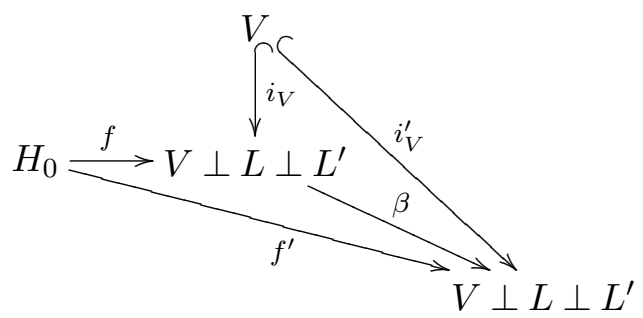

which implies that $\beta \circ i_{V}=i_{V}^{\prime}$. Thus, $\beta=\operatorname{Id}_{V} \perp \beta^{\prime}$, where $\beta^{\prime}: L \perp L^{\prime} \rightarrow L \perp L^{\prime}$ is a morphism in $\operatorname{Hom}_{\mathcal{E}_{q}}\left(L \perp L^{\prime}, L \perp L^{\prime}\right)$. Consequently,

$f\left(a_{0}\right)=v+l, f\left(b_{0}\right)=w+l^{\prime} \quad$ and $\quad f^{\prime}\left(a_{0}\right)=v+\beta^{\prime}(l), f^{\prime}\left(b_{0}\right)=w+\beta^{\prime}\left(l^{\prime}\right)$,

and we deduce that $p_{V} \circ f=p_{V}^{\prime}$. Furthermore, since $\beta^{\prime}$ is invertible, for all $x$ in $L \perp L^{\prime}$ we have: $x$ is nonzero if and only if $\beta^{\prime}(x)$ is nonzero. Consequently, $T$ and $T^{\prime}$ have the same type. 
This proposition justifies the following notation.

Notation 3.6. We will denote by $A_{v, w}, B_{v, w}$ and $C_{v, w}$ the morphisms in $\operatorname{Hom}_{\mathcal{T}_{q}}\left(H_{0}, V\right)$ respectively of type $A, B$ and $C$ and such that $p_{V} \circ f\left(a_{0}\right)=v$ and $p_{V} \circ f\left(b_{0}\right)=w$.

The following result is a straightforward consequence of Lemma 3.2 and Proposition 3.4.

LEMMA 3.7. A basis of $P_{H_{0}}^{(1)} / P_{H_{0}}^{(0)}(V)$ is given by the set

$$
\begin{aligned}
\mathcal{B}_{H_{0}}^{(1)}= & \left\{\left[A_{v, w}\right] \mid v, w \in V, q(v)=0, B(v, w)=1\right\} \\
& \cup\left\{\left[B_{v, w}\right] \mid v, w \in V, q(w)=0, B(v, w)=1\right\} \\
& \cup\left\{\left[C_{v, w}\right] \mid v, w \in V, q(v+w)=1, B(v, w)=1\right\} .
\end{aligned}
$$

By Proposition 2.19, we have $\left(P_{H_{0}} / P_{H_{0}}^{(1)}\right)(V) \simeq \kappa\left(\right.$ iso $\left._{H_{0}}\right)(V)$. This yields the following result.

LEMMA 3.8. $A$ basis of $P_{H_{0}} / P_{H_{0}}^{(1)}(V)$ is given by the set

$$
\mathcal{B}_{H_{0}}^{(2)}=\left\{\left[D_{f}\right] \mid f \in \operatorname{Hom}_{\mathcal{E}_{q}}\left(H_{0}, V\right)\right\},
$$

where $D_{f}$ is the morphism of $\mathcal{T}_{q}$ represented by the diagram $H_{0} \stackrel{f}{\rightarrow} V \stackrel{\text { Id }}{\longleftarrow} V$.

We end this section by giving the composition rules for the morphisms $t_{f}$, $A_{v, w}, B_{v, w}, C_{v, w}$ and $D_{f}$. This technical result will be fundamental in the following section, to prove the splitting of the filtration.

Lemma 3.9. Let $T=V \stackrel{\varphi}{\longrightarrow} W \perp L \stackrel{i_{W}}{\longleftarrow} W$ be a morphism in $\operatorname{Hom}_{\mathcal{T}_{q}}(V, W)$.

(1) For $f \in \operatorname{Hom}_{\mathcal{E}^{f}}\left(\mathbb{F}_{2}{ }^{\oplus 2}, \epsilon(V)\right)$ we have

$$
T \circ t_{f}=t_{\varphi \circ f} .
$$

(2) (a) For $v, w \in V$ with $q(v)=0$ and $B(v, w)=1$, we have

$$
T \circ A_{v, w}= \begin{cases}A_{\varphi(v), p_{W} \circ \varphi(w)} & \text { if } \varphi(v) \in W, \\ t_{p_{W} \circ(\varphi \perp \mathrm{Id}) \circ \alpha} & \text { otherwise. }\end{cases}
$$

(b) For $v, w \in V$ with $q(w)=0$ and $B(v, w)=1$, we have

$$
T \circ B_{v, w}= \begin{cases}B_{p_{W} \circ \varphi(v), \varphi(w)} & \text { if } \varphi(w) \in W, \\ t_{p_{W} \circ(\varphi \perp \mathrm{Id}) \circ \alpha} & \text { otherwise. }\end{cases}
$$

(c) For $v, w \in V$ with $q(v+w)=1$ and $B(v, w)=1$, we have

$$
T \circ C_{v, w}= \begin{cases}C_{p_{W} \circ \varphi(v), p_{W} \circ \varphi(w)} & \text { if } \varphi(v+w) \in W, \\ t_{p_{W} \circ(\varphi \perp \mathrm{Id}) \circ \alpha} & \text { otherwise. }\end{cases}
$$


(3) For $f \in \operatorname{Hom}_{\mathcal{E}_{q}}\left(H_{0}, V\right)$, we have $T \circ D_{f}=$
$\left\{\begin{array}{l}D_{\varphi \circ f} \\ A_{\varphi \circ f\left(a_{0}\right), p_{W} \circ \varphi \circ f\left(b_{0}\right)} \\ B_{p_{W} \circ \varphi \circ f\left(a_{0}\right), \varphi \circ f\left(b_{0}\right)} \\ C_{p_{W} \circ \varphi \circ f\left(a_{0}\right), p_{W} \circ \varphi \circ f\left(b_{0}\right)} \\ t_{p_{W} \circ(\varphi \perp \mathrm{Id}) \circ \alpha}\end{array}\right.$

$$
\begin{aligned}
& \text { if } \varphi \circ f\left(a_{0}\right) \in W \text { and } \varphi \circ f\left(b_{0}\right) \in W, \\
& \text { if } \varphi \circ f\left(a_{0}\right) \in W \text { and } \varphi \circ f\left(b_{0}\right) \notin W, \\
& \text { if } \varphi \circ f\left(a_{0}\right) \notin W \text { and } \varphi \circ f\left(b_{0}\right) \in W, \\
& \text { if } \varphi \circ f\left(a_{0}\right) \notin W \text { and } \varphi \circ f\left(b_{0}\right) \notin W \\
& \text { and } \varphi \circ f\left(a_{0}+b_{0}\right) \in W, \\
& \text { if } \varphi \circ f\left(a_{0}\right) \notin W \text { and } \varphi \circ f\left(b_{0}\right) \notin W \\
& \text { and } \varphi \circ f\left(a_{0}+b_{0}\right) \notin W \text {. }
\end{aligned}
$$

Proof. By definition of the composition in $\mathcal{T}_{q}$, we have the following diagram:

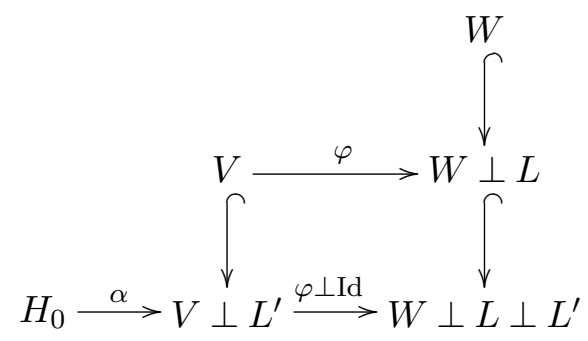

(1) For a morphism $t_{f}$, we have $\alpha\left(a_{0}\right)=f\left(a_{0}\right)+l$ and $\alpha\left(b_{0}\right)=f\left(b_{0}\right)+m$, where $\{l, m\}$ is a linearly independent set in $L^{\prime}$. Consequently,

$$
(\varphi \perp \mathrm{Id}) \circ \alpha\left(a_{0}\right)=\varphi \circ f\left(a_{0}\right)+l \quad \text { and } \quad(\varphi \perp \mathrm{Id}) \circ \alpha\left(b_{0}\right)=\varphi \circ f\left(b_{0}\right)+m \text {, }
$$

proving that $T \circ t_{f}=t_{\varphi \circ f}$.

(2) For $A_{v, w}$, we have $\alpha\left(a_{0}\right)=v$ and $\alpha\left(b_{0}\right)=w+l^{\prime}$, where $l^{\prime}$ is a nonzero element of $L^{\prime}$. Consequently,

$$
(\varphi \perp \mathrm{Id}) \circ \alpha\left(a_{0}\right)=\varphi(v) \quad \text { and } \quad(\varphi \perp \mathrm{Id}) \circ \alpha\left(b_{0}\right)=\varphi(w)+l^{\prime} .
$$

We have to distinguish two cases:

- If $\varphi(v) \in W$, then since $\varphi$ preserves quadratic forms, we have $q(\varphi(v))=q(v)$, and since $L^{\prime}$ is orthogonal to $V, B\left(\varphi(v), p_{W} \circ \varphi(w)\right)=$ $B(\varphi(v), \varphi(w))=B(v, w)$. Thus the morphism $A_{\varphi(v), p_{W} \circ \varphi(w)}$ is defined and we have $T \circ A_{v, w}=A_{\varphi(v), p_{W} \circ \varphi(w)}$.

- Otherwise, $\varphi(v)=p_{W} \circ \varphi(v)+m$, where $m$ is a nonzero element of $L$. Consequently, we obtain a morphism of null rank and $T \circ A_{v, w}=$ $t_{p_{W} \circ(\varphi \perp \mathrm{Id}) \circ \alpha}$.

The cases of $B_{v, w}$ and $C_{v, w}$ are similar and are left to the reader.

(3) For the morphism $D_{f}$, where $f \in \operatorname{Hom}_{\mathcal{E}_{q}}\left(H_{0}, V\right)$, we have $\alpha\left(a_{0}\right)=$ $f\left(a_{0}\right)=v$ and $\alpha\left(b_{0}\right)=f\left(b_{0}\right)=w$ for some $v, w \in V$. Consequently, $(\varphi \perp$ Id $) \circ$ $\alpha\left(a_{0}\right)=\varphi(v)$ and $(\varphi \perp \mathrm{Id}) \circ \alpha\left(b_{0}\right)=\varphi(w)$. Since $\varphi \circ f$ preserves the quadratic 
forms, we have $q\left(\varphi \circ f\left(a_{0}\right)\right)=q\left(\varphi \circ f\left(b_{0}\right)\right)=0$ and $B\left(\varphi \circ f\left(a_{0}\right), \varphi \circ f\left(b_{0}\right)\right)=1$. Thus the morphisms $A_{\varphi \circ f\left(a_{0}\right), \varphi \circ f\left(b_{0}\right)}, B_{\varphi \circ f\left(a_{0}\right), \varphi \circ f\left(b_{0}\right)}$ and $C_{\varphi \circ f\left(a_{0}\right), \varphi \circ f\left(b_{0}\right)}$ are defined.

We have to distinguish four cases:

- If $\varphi(v) \in W$ and $\varphi(w) \in W$ then $T \circ D_{f}=D_{\varphi \circ f}$.

- If $\varphi \circ f\left(a_{0}\right) \in W$ and $\varphi \circ f\left(b_{0}\right) \notin W$, then $\varphi \circ f\left(a_{0}\right)=w^{\prime}$ and $\varphi \circ f\left(b_{0}\right)=$ $w^{\prime \prime}+l$, where $l \in L \backslash\{0\}$. Consequently, we obtain a morphism of type $A$ and $T \circ D_{f}=A_{\varphi \circ f\left(a_{0}\right), \varphi \circ f\left(b_{0}\right)}$.

- If $\varphi \circ f\left(a_{0}\right) \notin W$ and $\varphi \circ f\left(b_{0}\right) \in W$, then $\varphi \circ f\left(a_{0}\right)=w^{\prime}+l$ and $\varphi \circ f\left(b_{0}\right)=w^{\prime \prime}$, where $l \in L \backslash\{0\}$. Consequently, we obtain a morphism of type $B$ and $T \circ D_{f}=B_{\varphi \circ f\left(a_{0}\right), \varphi \circ f\left(b_{0}\right)}$.

- If $\varphi \circ f\left(a_{0}\right) \notin W, \varphi \circ f\left(b_{0}\right) \notin W$ and $\varphi \circ f\left(a_{0}+b_{0}\right) \in W$, then $\varphi \circ f\left(a_{0}\right)=w^{\prime}+l$ and $\varphi \circ f\left(b_{0}\right)=w^{\prime \prime}+l$, where $l \in L \backslash\{0\}$. Consequently, we obtain a morphism of type $C$ and $T \circ D_{f}=C_{\varphi \circ f\left(a_{0}\right), \varphi \circ f\left(b_{0}\right)}$.

- If $\varphi \circ f\left(a_{0}\right) \notin W, \varphi \circ f\left(b_{0}\right) \notin W$ and $\varphi \circ f\left(a_{0}+b_{0}\right) \notin W$, then $\varphi \circ f\left(a_{0}\right)=$ $w^{\prime}+l$ and $\varphi \circ f\left(b_{0}\right)=w^{\prime \prime}+l^{\prime}$, where $l, l^{\prime} \in L \backslash\{0\}$. Consequently, we obtain a morphism of null rank and $T \circ D_{f}=t_{p_{W} \circ(\varphi \perp \text { Id }) \circ \alpha}$

3.1.2. Splitting of the filtration for the functor $P_{H_{0}}$. In this section, we prove the following result.

Proposition 3.10. The rank filtration splits for the functor $P_{H_{0}}$, namely

$$
P_{H_{0}}=P_{H_{0}}^{(0)} \oplus P_{H_{0}}^{(1)} / P_{H_{0}}^{(0)} \oplus P_{H_{0}} / P_{H_{0}}^{(1)} .
$$

Proof. By Theorem 2.6, we have $P_{H_{0}}^{(1)}=P_{H_{0}}^{(0)} \oplus P_{H_{0}}^{(1)} / P_{H_{0}}^{(0)}$. To prove the proposition, it is sufficient to prove that $P_{H_{0}}=P_{H_{0}}^{(1)} \oplus P_{H_{0}} / P_{H_{0}}^{(1)}$.

By definition of the filtration, we have the short exact sequence

$$
0 \rightarrow P_{H_{0}}^{(1)} \rightarrow P_{H_{0}} \stackrel{p}{\rightarrow} P_{H_{0}} / P_{H_{0}}^{(1)} \rightarrow 0 .
$$

Let $V$ be an object in $\mathcal{T}_{q}$, and consider a morphism $f \in \operatorname{Hom}_{\mathcal{E}_{q}}\left(H_{0}, V\right)$ and the generator $\left[D_{f}\right]$ of $P_{H_{0}} / P_{H_{0}}^{(1)}(V)$ associated to $f$. Since $f$ preserves the quadratic forms, we have $q\left(f\left(a_{0}\right)\right)=q\left(f\left(b_{0}\right)\right)=0, q\left(f\left(a_{0}+b_{0}\right)\right)=1$; thus $B\left(f\left(a_{0}\right), f\left(b_{0}\right)\right)=1$. Consequently, the morphisms $A_{f\left(a_{0}\right), f\left(b_{0}\right)}, B_{f\left(a_{0}\right), f\left(b_{0}\right)}$ and $C_{f\left(a_{0}\right), f\left(b_{0}\right)}$ in $\operatorname{Hom}_{\mathcal{T}_{q}}\left(H_{0}, V\right)$ are defined. We define a map $s_{V}$ : $P_{H_{0}} / P_{H_{0}}^{(1)}(V) \rightarrow P_{H_{0}}(V)$ by

$$
\begin{aligned}
s_{V}: P_{H_{0}} / P_{H_{0}}^{(1)}(V) & \rightarrow P_{H_{0}}(V), \\
{\left[D_{f}\right] } & \mapsto\left[D_{f}\right]+\left[A_{f\left(a_{0}\right), f\left(b_{0}\right)}\right]+\left[B_{f\left(a_{0}\right), f\left(b_{0}\right)}\right]+\left[C_{f\left(a_{0}\right), f\left(b_{0}\right)}\right] .
\end{aligned}
$$

We verify the following two statements. 
(1) $p_{V} \circ s_{V}=\mathrm{Id}$. Indeed, for any canonical generator $\left[D_{f}\right]$ of $P_{H_{0}} / P_{H_{0}}^{(1)}(V)$, we have

$$
p_{V} \circ s_{V}\left(\left[D_{f}\right]\right)=\left[D_{f}\right]
$$

since the morphisms $A_{f\left(a_{0}\right), f\left(b_{0}\right)}, B_{f\left(a_{0}\right), f\left(b_{0}\right)}$ and $C_{f\left(a_{0}\right), f\left(b_{0}\right)}$ have rank one.

(2) The maps $s_{V}$ define a natural map. Indeed, for a morphism $T=$ $V \stackrel{\varphi}{\rightarrow} W \perp L \stackrel{i_{W}}{\longleftarrow} W$ in $\operatorname{Hom}_{\mathcal{T}_{q}}(V, W)$, we have the commutative diagram

$$
\begin{gathered}
P_{H_{0}} / P_{H_{0}}^{(1)}(V) \stackrel{s_{V}}{\longrightarrow} P_{H_{0}}(V) \\
P_{H_{0}} / P_{H_{0}}^{(1)}(T){ }^{\downarrow} \\
P_{H_{0}} / P_{H_{0}}^{(1)}(W) \stackrel{s_{W}}{\longrightarrow} P_{H_{0}}(W)
\end{gathered}
$$

To simplify notation, write

$$
\begin{aligned}
A^{\prime} & =A_{\varphi \circ f\left(a_{0}\right), p_{W} \circ \varphi \circ f\left(b_{0}\right)}, & B^{\prime} & =B_{p_{W} \circ \varphi \circ f\left(a_{0}\right), \varphi \circ f\left(b_{0}\right)}, \\
C^{\prime} & =C_{p_{W} \circ \varphi \circ f\left(a_{0}\right), p_{W} \circ \varphi \circ f\left(b_{0}\right)}, & t^{\prime} & =t_{p_{W} \circ(\varphi \perp \mathrm{Id}) \circ \alpha} .
\end{aligned}
$$

On the one hand, by Lemma 3.9,

$$
\begin{aligned}
P_{H_{0}}(T) \circ s_{V}\left(\left[D_{f}\right]\right) & =P_{H_{0}}(T)\left(\left[D_{f}\right]+\left[A_{f\left(a_{0}\right), f\left(b_{0}\right)}\right]+\left[B_{f\left(a_{0}\right), f\left(b_{0}\right)}\right]+\left[C_{f\left(a_{0}\right), f\left(b_{0}\right)}\right]\right) \\
= & {\left[T \circ D_{f}\right]+\left[T \circ A_{f\left(a_{0}\right), f\left(b_{0}\right)}\right]+\left[T \circ B_{\left.f\left(a_{0}\right), f\left(b_{0}\right)\right]+\left[T \circ C_{f\left(a_{0}\right), f\left(b_{0}\right)}\right]}\right.} \\
& \begin{cases}{\left[D_{\varphi \circ f}\right]+\left[A^{\prime}\right]+\left[B^{\prime}\right]+\left[C^{\prime}\right]} & \text { if } \varphi \circ f\left(a_{0}\right) \in W \text { and } \varphi \circ f\left(b_{0}\right) \in W, \\
{\left[A^{\prime}\right]+\left[A^{\prime}\right]+\left[t^{\prime}\right]+\left[t^{\prime}\right]=0} & \text { if } \varphi \circ f\left(a_{0}\right) \in W \text { and } \varphi \circ f\left(b_{0}\right) \notin W, \\
{\left[B^{\prime}\right]+\left[t^{\prime}\right]+\left[B^{\prime}\right]+\left[t^{\prime}\right]=0} & \text { if } \varphi \circ f\left(a_{0}\right) \notin W \text { and } \varphi \circ f\left(b_{0}\right) \in W, \\
{\left[C^{\prime}\right]+\left[t^{\prime}\right]+\left[t^{\prime}\right]+\left[C^{\prime}\right]=0} & \text { if } \varphi \circ f\left(a_{0}\right) \notin W \text { and } \varphi \circ f\left(b_{0}\right) \notin W \\
{\left[t^{\prime}\right]+\left[t^{\prime}\right]+\left[t^{\prime}\right]+\left[t^{\prime}\right]=0} & \text { and } \varphi \circ f\left(a_{0}+b_{0}\right) \in W, \\
= & \text { if } \varphi \circ f\left(a_{0}\right) \notin W \text { and } \varphi \circ f\left(b_{0}\right) \notin W \\
{\left[D_{\varphi \circ f}\right]+\left[A^{\prime}\right]+\left[B^{\prime}\right]+\left[C^{\prime}\right]} & \text { if } \varphi \circ f\left(a_{0}\right) \in W \text { and } \varphi \circ f\left(b_{0}\right) \in W, \\
0 & \text { otherwise. }\end{cases}
\end{aligned}
$$

On the other hand, by Lemma 3.9,

$$
P_{H_{0}} / P_{H_{0}}^{(1)}(T)\left(\left[D_{f}\right]\right)= \begin{cases}{\left[D_{\varphi \circ f}\right]} & \text { if } \varphi \circ f\left(a_{0}\right) \in W \text { and } \varphi \circ f\left(b_{0}\right) \in W, \\ 0 & \text { otherwise, }\end{cases}
$$

since the morphisms $A, B, C$ and $t$ are zero in the quotient $P_{H_{0}} / P_{H_{0}}^{(1)}(W)$. Hence,

$$
s_{W} \circ P_{H_{0}} / P_{H_{0}}^{(1)}(T)\left(\left[D_{f}\right]\right)= \begin{cases}{\left[D_{\varphi \circ f}\right]+\left[A^{\prime}\right]+\left[B^{\prime}\right]+\left[C^{\prime}\right]} & \text { if } \varphi \circ f\left(a_{0}\right) \in W \\ 0 & \text { and } \varphi \circ f\left(b_{0}\right) \in W, \\ 0 & \text { otherwise }\end{cases}
$$


Consequently, the maps $s_{V}$ define a natural map which is a section of $p$. This gives rise to the splitting of the exact sequence (3.10.1).

3.1.3. Identification of the direct summands. The aim of this section is to identify the summands of the decomposition in Proposition 3.10. We begin by proving that the morphisms of type $A$ (respectively of type $B$ and $C$ ) define a subfunctor of $P_{H_{0}}^{(1)} / P_{H_{0}}^{(0)}$ which is a direct summand of this functor.

Lemma 3.11. The functor $P_{H_{0}}^{(1)} / P_{H_{0}}^{(0)}$ admits the following decomposition into direct summands:

$$
P_{H_{0}}^{(1)} / P_{H_{0}}^{(0)}=F_{A} \oplus F_{B} \oplus F_{C},
$$

where $F_{A}, F_{B}$ and $F_{C}$ are subfunctors of $P_{H_{0}}^{(1)} / P_{H_{0}}^{(0)}$ generated by, respectively, the morphisms of type $A, B$ and $C$.

Proof. By Lemma 3.7, we have an isomorphism of vector spaces

$$
P_{H_{0}}^{(1)} / P_{H_{0}}^{(0)}(V)=F_{A}(V) \oplus F_{B}(V) \oplus F_{C}(V),
$$

for all objects $V$ in $\mathcal{T}_{q}$. Consequently, it is sufficient to prove that $F_{A}, F_{B}$ and $F_{C}$ are subfunctors of $P_{H_{0}}^{(1)} / P_{H_{0}}^{(0)}$.

For $F_{A}$, we have to verify the commutativity of the diagram

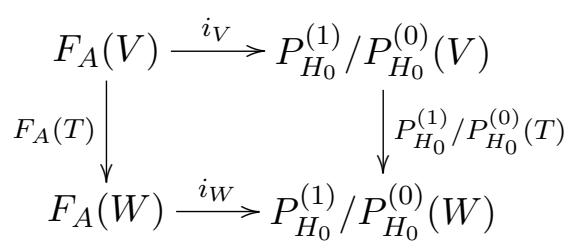

where $T \in \operatorname{Hom}_{\mathcal{T}_{q}}(V, W)$. Let $\left[A_{v, w}\right]$ be a canonical generator of $F_{A}(V)$. By Lemma 3.9 we have

$$
T \circ A_{v, w}= \begin{cases}A_{\varphi(v), p_{W} \circ \varphi(w)} & \text { if } \varphi(v) \in W \\ t_{p_{W} \circ(\varphi \perp \text { Id }) \circ \alpha} & \text { otherwise. }\end{cases}
$$

Consequently,

$$
P_{H_{0}}^{(1)} / P_{H_{0}}^{(0)}(T) \circ i_{V}\left(\left[A_{v, w}\right]\right)= \begin{cases}{\left[A_{\varphi(v), p_{W} \circ \varphi(w)}\right]} & \text { if } \varphi(v) \in W \\ 0 & \text { otherwise }\end{cases}
$$

since the morphism $t_{p_{W} \circ(\varphi \perp \text { Id }) \circ \alpha}$ has null rank. We see that $P_{H_{0}}^{(1)} / P_{H_{0}}^{(0)}(T) \circ$ $i_{V}\left(\left[A_{v, w}\right]\right)$ is in the vector space $F_{A}(W)$; thus, $F_{A}$ is a subfunctor of $P_{H_{0}}^{(1)} / P_{H_{0}}^{(0)}$.

In the same way, by the use of the values of the $T \circ B_{v, w}$ and $T \circ C_{v, w}$ given in Lemma 3.9, we prove that $F_{B}$ and $F_{C}$ are subfunctors of $P_{H_{0}}^{(1)} / P_{H_{0}}^{(0)}$.

In the following lemma, we identify the functors $F_{A}, F_{B}$ and $F_{C}$ with certain mixed functors defined in [11] and recalled in Section 1. 
LEMMA 3.12.

(1) $F_{A}$ and $F_{B}$ are isomorphic to the functor $\operatorname{Mix}_{0,1}$.

(2) $F_{C}$ is isomorphic to $\mathrm{Mix}_{1,1}$.

Proof. To prove $F_{A} \simeq \operatorname{Mix}_{0,1}$, let $\left[A_{v, w}\right]$ be a canonical generator of $F_{A}(V)$. By definition, $B(v, w)=1$. Consequently, the following linear map exists:

$$
\sigma_{V}^{1}: F_{A}(V) \rightarrow \operatorname{Mix}_{0,1}(V), \quad\left[A_{v, w}\right] \mapsto[(w, v+w)] .
$$

It is an isomorphism with inverse

$$
\left(\sigma_{V}^{1}\right)^{-1}: \operatorname{Mix}_{0,1}(V) \rightarrow F_{A}(V), \quad[(v, w)] \mapsto\left[A_{v+w, v}\right] .
$$

We have to verify that the maps $\sigma_{V}^{1}$ define a natural map, that is, for a morphism $T=\left[V \stackrel{\varphi}{\longrightarrow} W \perp L \stackrel{i_{W}}{\longleftarrow} W\right]$, the following diagram is commutative:

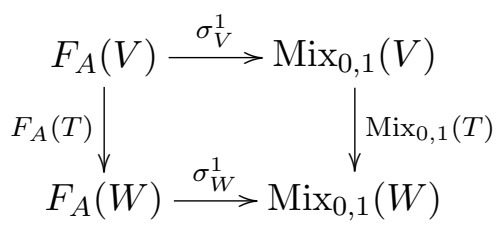

We have

$$
\begin{aligned}
\operatorname{Mix}_{0,1}(T) \circ \sigma_{V}^{1}\left(\left[A_{v, w}\right]\right) & =\operatorname{Mix}_{0,1}(T)[(w, v+w)] \\
& = \begin{cases}{\left[\left(p_{W} \circ \varphi(w), p_{W} \circ \varphi(v+w)\right)\right]} & \text { if } \varphi(v) \in W, \\
0 & \text { otherwise, }\end{cases}
\end{aligned}
$$

by the definition of mixed functors, and

$$
\begin{aligned}
\sigma_{W}^{1} \circ F_{A}(T)\left(\left[A_{v, w}\right]\right) & =\sigma_{W}^{1} \begin{cases}{\left[A_{\varphi(v), p_{W} \circ \varphi(w)}\right]} & \text { if } \varphi(v) \in W, \\
0 & \text { otherwise, }\end{cases} \\
& = \begin{cases}{\left[\left(p_{W} \circ \varphi(w), \varphi(v)+p_{W} \circ \varphi(w)\right)\right]} & \text { if } \varphi(v) \in W, \\
0 & \text { otherwise. }\end{cases}
\end{aligned}
$$

When $\varphi(v) \in W$, we have

$$
\left[\left(p_{W} \circ \varphi(w), \varphi(v)+p_{W} \circ \varphi(w)\right)\right]=\left[\left(p_{W} \circ \varphi(w), p_{W} \circ \varphi(v+w)\right)\right],
$$

which proves the naturality of $\sigma^{1}$.

Since the other two cases are similar, we only give the definition of the relevant isomorphism of vector spaces and leave it to the reader to verify that they define natural equivalences.

For $F_{B} \simeq \operatorname{Mix}_{1,0}$, the map is

$$
\sigma_{V}^{2}: F_{B}(V) \rightarrow \operatorname{Mix}_{0,1}(V), \quad B_{v, w} \mapsto[(v, v+w)],
$$

and for $F_{C} \simeq \operatorname{Mix}_{1,1}$, it is

$$
\sigma_{V}^{3}: F_{C}(V) \rightarrow \operatorname{Mix}_{1,1}(V), \quad C_{v, w} \mapsto[(v, w)] .
$$


Proposition 3.13. The projective functor $P_{H_{0}}$ admits the following decomposition into direct summands:

$$
P_{H_{0}}=\iota\left(P_{\epsilon\left(\mathbb{F}_{2} \oplus 2\right)}^{\mathcal{F}}\right) \oplus\left(\operatorname{Mix}_{0,1} \oplus 2 \oplus \operatorname{Mix}_{1,1}\right) \oplus \kappa\left(\operatorname{iso}_{H_{0}}\right),
$$

where $\operatorname{Mix}_{0,1}$ and $\operatorname{Mix}_{1,1}$ are mixed functors and iso $_{H_{0}}$ is an isotropic functor.

Proof. This is a straightforward consequence of Proposition 3.10, Theorem 2.6, Proposition 2.19 and Lemmas 3.11 and 3.12.

3.2. Decomposition of $P_{H_{1}}$. The study of the functor $P_{H_{1}}$ is analogous to that of $P_{H_{0}}$ in the previous section. Therefore, we only give the principal results without proofs.

3.2.1. Explicit description of the subquotients of the filtration. In this section, we give bases of the vector spaces $P_{H_{1}}^{(0)}(V), P_{H_{1}}^{(1)} / P_{H_{1}}^{(0)}(V)$ and $P_{H_{1}} / P_{H_{1}}^{(1)}(V)$ for $V$ an object in $\mathcal{T}_{q}$.

Lemma 3.14. A basis of $P_{H_{1}}^{(0)}(V)$ is given by the set

$$
\mathcal{B}_{H_{1}}^{(0)}=\left\{t_{f} \mid f \in \operatorname{Hom}_{\mathcal{E}^{f}}\left(\mathbb{F}_{2}{ }^{\oplus 2}, \epsilon(V)\right)\right\} .
$$

LEMMA 3.15. Let $T=\left[H_{1} \stackrel{f}{\rightarrow} V \perp L \stackrel{i_{V}}{\longleftarrow} V\right]$ be a morphism of $\mathcal{T}_{q}$ which represents a canonical generator of $P_{H_{1}}^{(1)} / P_{H_{1}}^{(0)}(V)$, and $\left\{a_{1}, b_{1}\right\}$ a symplectic basis of $H_{1}$. Then the map $f$ in $T$ has one of the following three forms.

(1) If $I=\left(f\left(a_{1}\right), 0\right)$ then $f: H_{1} \rightarrow V \perp L$ is defined by

$$
f\left(a_{1}\right)=v \quad \text { and } \quad f\left(b_{1}\right)=w+l
$$

for some $v, w \in V$ satisfying $q(v)=1$ and $B(v, w)=1$, and some $l \in L \backslash\{0\}$.

(2) If $I=\left(f\left(b_{1}\right), 0\right)$ then $f: H_{1} \rightarrow V \perp L$ is defined by

$$
f\left(a_{1}\right)=v+l \quad \text { and } \quad f\left(b_{1}\right)=w
$$

for some $v, w \in V$ satisfying $q(w)=1$ and $B(v, w)=1$, and some $l \in L \backslash\{0\}$.

(3) If $I=\left(f\left(a_{1}+b_{1}\right), 1\right)$ then $f: H_{1} \rightarrow V \perp L$ is defined by

$$
f\left(a_{1}\right)=v+l \quad \text { and } \quad f\left(b_{1}\right)=w+l
$$

for some $v, w \in V$ satisfying $q(v+w)=1$ and $B(v, w)=1$, and some $l \in L \backslash\{0\}$.

Notation 3.16. The morphisms $\left[H_{1} \stackrel{f}{\rightarrow} V \perp L \stackrel{i_{V}}{\longleftarrow} V\right]$, where $f$ is as in item (1) (respectively (2) and (3)) of the previous lemma will be referred to as type $E$ (respectively $F$ and $G$ ) morphisms. 
The proposition analogous to Proposition 3.4 holds for $H_{1}$. This justifies the following notation.

Notation 3.17. Denote by $E_{v, w}, F_{v, w}$ and $G_{v, w}$ the morphisms in $\operatorname{Hom}_{\mathcal{T}_{q}}\left(H_{1}, V\right)$ respectively of type $E, F$ and $G$ such that $p_{V} \circ f\left(a_{1}\right)=v$ and $p_{V} \circ f\left(b_{1}\right)=w$.

We deduce the following lemmas.

Lemma 3.18. A basis of $P_{H_{1}}^{(1)} / P_{H_{1}}^{(0)}(V)$ is given by the set

$$
\begin{aligned}
\mathcal{B}_{H_{1}}^{(1)}= & \left\{\left[E_{v, w}\right] \mid v, w \in V, q(v)=1, B(v, w)=1\right\} \\
& \cup\left\{\left[F_{v, w}\right] \mid v, w \in V, q(w)=1, B(v, w)=1\right\} \\
& \cup\left\{\left[G_{v, w}\right] \mid v, w \in V, q(v+w)=1, B(v, w)=1\right\} .
\end{aligned}
$$

Lemma 3.19. A basis of $P_{H_{1}} / P_{H_{1}}^{(1)}(V)$ is given by the set

$$
\mathcal{B}_{H_{1}}^{(2)}=\left\{\left[H_{f}\right] \mid f \in \operatorname{Hom}_{\mathcal{E}_{q}}\left(H_{1}, V\right)\right\},
$$

where $H_{f}$ is the morphism of $\mathcal{T}_{q}$ represented by the diagram $H_{1} \stackrel{f}{\rightarrow} V \stackrel{\text { Id }}{\longleftarrow} V$.

The composition rules for $E_{v, w}, F_{v, w}, G_{v, w}$ and $H_{f}$ are similar to those for $A_{v, w}, B_{v, w}, C_{v, w}$ and $D_{f}$, given in Lemma 3.9. The details can be provided by the reader.

3.2.2. Splitting of the filtration for $P_{H_{1}}$

PRoposition 3.20. The rank filtration splits for the functor $P_{H_{1}}$, namely

$$
P_{H_{1}}=P_{H_{1}}^{(0)} \oplus P_{H_{1}}^{(1)} / P_{H_{1}}^{(0)} \oplus P_{H_{1}} / P_{H_{1}}^{(1)} .
$$

Proof. One verifies that the map $s_{V}: P_{H_{1}} / P_{H_{1}}^{(1)}(V) \rightarrow P_{H_{1}}(V)$ given by

$$
\begin{aligned}
s_{V}: P_{H_{1}} / P_{H_{1}}^{(1)}(V) & \rightarrow P_{H_{1}}(V), \\
{\left[H_{f}\right] } & \mapsto\left[H_{f}\right]+\left[E_{f\left(a_{1}\right), f\left(b_{1}\right)}\right]+\left[F_{f\left(a_{1}\right), f\left(b_{1}\right)}\right]+\left[G_{f\left(a_{1}\right), f\left(b_{1}\right)}\right],
\end{aligned}
$$

defines a natural map which is a section of the projection $P_{H_{1}} \rightarrow P_{H_{1}} / P_{H_{1}}^{(1)}$.

3.2.3. Identification of the direct summands

LEMma 3.21. The functor $P_{H_{1}}^{(1)} / P_{H_{1}}^{(0)}$ admits the following decomposition into direct summands:

$$
P_{H_{1}}^{(1)} / P_{H_{1}}^{(0)}=F_{E} \oplus F_{F} \oplus F_{G},
$$

where $F_{E}, F_{F}$ and $F_{G}$ are subfunctors of $P_{H_{1}}^{(1)} / P_{H_{1}}^{(0)}$ generated by, respectively, the morphisms of type $E, F$ and $G$.

Lemma 3.22. The functors $F_{E}, F_{F}$ and $F_{G}$ are equivalent to the functor $\operatorname{Mix}_{1,1}$. 
Proposition 3.23. The projective functor $P_{H_{1}}$ admits the following decomposition into direct summands:

$$
P_{H_{1}}=\iota\left(P_{\epsilon\left(\mathbb{F}_{2} \oplus 2\right.}^{\mathcal{F}}\right) \oplus \operatorname{Mix}_{1,1} \oplus 3 \oplus \kappa\left(\text { iso }_{H_{1}}\right) .
$$

3.3. Consequences of the decompositions of $P_{H_{0}}$ and $P_{H_{1}}$. In this section, we draw the conclusions of the decompositions of $P_{H_{0}}$ and $P_{H_{1}}$ given in Propositions 3.13 and 3.23. We deduce the indecomposability of the functors $\operatorname{Mix}_{0,1}$ and $\operatorname{Mix}_{1,1}$, we study the projectivity of the first isotropic functors in $\mathcal{F}_{\text {quad }}$, and we give a classification of the "small" simple objects of $\mathcal{F}_{\text {quad }}$.

3.3.1. Indecomposability of $\mathrm{Mix}_{0,1}$ and $\mathrm{Mix}_{1,1}$. The aim of this section is to prove the following result:

Proposition 3.24. The functors $\mathrm{Mix}_{0,1}$ and $\mathrm{Mix}_{1,1}$ are indecomposable.

The proof of this proposition relies on the following obvious lemma.

Lemma 3.25. If the functor $F$ in $\mathcal{F}_{\text {quad }}$ decomposes as a direct sum $F_{1} \oplus \cdots \oplus F_{n}$, then the projections $\pi_{i}: F \rightarrow F_{i}$ and the inclusions $j_{i}: F_{i} \rightarrow F$ induce idempotents $e_{i}=j_{i} \circ \pi_{i}$ in the ring $\operatorname{End}(F)$.

Proof of Proposition 3.24. By the Yoneda lemma,

$$
\operatorname{Hom}\left(P_{H_{0}}, \operatorname{Mix}_{0,1}\right)=\operatorname{Mix}_{0,1}\left(H_{0}\right) .
$$

By a calculation, the dimension of $\operatorname{Mix}_{0,1}\left(H_{0}\right)$ is 4 . According to Proposition 3.13, the multiplicity of the summand $\mathrm{Mix}_{0,1}$ in the decomposition of $P_{H_{0}}$ is 2. Consequently, the dimension of the vector space $E:=$ $\operatorname{Hom}\left(\operatorname{Mix}_{0,1}, \operatorname{Mix}_{0,1}\right)$ is 2 . We have the following basis: $\{\mathrm{Id}, \tau\}$, where $\tau([(u, v)])=[(v, u)]$. Consequently, $E=(\{0, \mathrm{Id}, \tau, \mathrm{Id}+\tau\},+, \circ)$ as a ring, and it is easy to see that this ring does not admit a nontrivial idempotent.

Similarly, $\operatorname{Hom}\left(P_{H_{0}}, \operatorname{Mix}_{1,1}\right)=\operatorname{Mix}_{1,1}\left(H_{0}\right)$ and $\operatorname{dim}\left(\operatorname{Mix}_{1,1}\left(H_{0}\right)\right)=2$. The multiplicity of the summand $\mathrm{Mix}_{1,1}$ in the decomposition of $P_{H_{0}}$ is 1 . We conclude that the ring $\operatorname{Hom}\left(\operatorname{Mix}_{1,1}, \operatorname{Mix}_{1,1}\right)$ does not admit a nontrivial idempotent.

We deduce from this proposition the following result, which complements Theorem 1.9, obtained in [11]:

Corollary 3.26. The short exact sequence $0 \rightarrow \Sigma_{\alpha, 1} \rightarrow \operatorname{Mix}_{\alpha, 1} \rightarrow$ $\Sigma_{\alpha, 1} \rightarrow 0$ does not split.

3.3.2. Projectivity of certain isotropic functors in $\mathcal{F}_{\text {quad }}$. The decompositions given in Propositions 3.13 and 3.23 allow us to study the projectivity of isotropic functors in $\mathcal{F}_{\text {quad }}$. Corollary 4.37 in [12] shows that the set of functors $\left\{\right.$ iso $\left._{V} \mid V \in \mathcal{S}\right\}$ is a set of projective generators of $\mathcal{F}_{\text {iso }}$, where $\mathcal{S}$ is a set of representatives of isometry classes of (possibly degenerate) quadratic spaces. 
Since the functor $\kappa\left(\right.$ iso $\left._{H_{0}}\right)$ (respectively $\kappa\left(\right.$ iso $\left.\left._{H_{1}}\right)\right)$ is a direct summand of $P_{H_{0}}$ (respectively $P_{H_{1}}$ ), we have the following result.

Proposition 3.27. The functors $\kappa\left(\right.$ iso $\left._{H_{0}}\right)$ and $\kappa\left(\right.$ iso $\left._{H_{1}}\right)$ are projective in the category $\mathcal{F}_{\text {quad }}$.

Corollary 1.6 and the previous proposition yield the following result.

COROLlaRY 3.28. The category $\mathcal{F}_{\text {quad }}$ contains nonconstant, finite, projective objects.

This is a new feature of the category $\mathcal{F}_{\text {quad }}$ compared with $\mathcal{F}$. Recall that, according to Corollary B7 in [5], due to Lionel Schwartz, the category $\mathcal{F}$ does not contain nonconstant finite projective functors.

Recall that the functor $\kappa\left(\operatorname{iso}_{(x, 0)}\right)$ is the top composition factor of $\operatorname{Mix}_{0,1}$, and $\kappa\left(\operatorname{iso}_{(x, 1)}\right)$ is that of $\operatorname{Mix}_{1,1}$. We have the following result.

Proposition 3.29. The projective cover of $\kappa\left(\mathrm{iso}_{(x, 0)}\right)$ (respectively $\left.\kappa\left(\operatorname{iso}_{(x, 1)}\right)\right)$ is the functor $\operatorname{Mix}_{0,1}$ (respectively $\left.\mathrm{Mix}_{1,1}\right)$. In particular, the functors $\kappa\left(\operatorname{iso}_{(x, 0)}\right)$ and $\kappa\left(\operatorname{iso}_{(x, 1)}\right)$ are not projective in $\mathcal{F}_{\text {quad }}$.

Proof. Since $\kappa\left(\operatorname{iso}_{(x, 0)}\right)\left(H_{0}\right) \neq\{0\}$ and $\kappa\left(\right.$ iso $\left._{(x, 1)}\right)\left(H_{0}\right) \neq\{0\}$, if these two functors were projective, they would be direct summands of $P_{H_{0}}$. We deduce from Proposition 3.13 that these functors are not projective.

Remark 3.30. Propositions 3.27 and 3.29 suggest the conjecture that, for a nondegenerate $\mathbb{F}_{2}$-quadratic space $H, \kappa\left(\right.$ iso $\left._{H}\right)$ is a projective functor in $\mathcal{F}_{\text {quad }}$ and, for a degenerate quadratic space $D, \kappa\left(\right.$ iso $\left._{D}\right)$ is not a projective functor in $\mathcal{F}_{\text {quad }}$ and its projective cover is a generalized mixed functor. This will be the subject of future work.

3.3.3. Classification of simple objects $S$ of $\mathcal{F}_{\text {quad }}$ such that either $S\left(H_{0}\right) \neq 0$ or $S\left(H_{1}\right) \neq 0$. If $S$ is a simple object in $\mathcal{F}_{\text {quad }}$ such that $S\left(H_{0}\right) \neq 0$, the Yoneda lemma implies that $\operatorname{Hom}\left(P_{H_{0}}, S\right)=S\left(H_{0}\right) \neq 0$. Consequently, there exists a morphism of $\mathcal{F}_{\text {quad }}$ from $P_{H_{0}}$ to $S$ which is an epimorphism, by simplicity of $S$. From the decompositions given in Propositions 3.13 and 3.23, from Corollary 1.7 concerning the functors iso $H_{0}$ and iso $_{H_{1}}$ and from the study of the functors $\mathrm{Mix}_{0,1}$ and $\mathrm{Mix}_{1,1}$ done in [11] and recalled in Section 1, we deduce the following result.

Proposition 3.31. The isomorphism classes of nonconstant simple functors in $\mathcal{F}_{\text {quad }}$ such that either $S\left(H_{0}\right) \neq 0$ or $S\left(H_{1}\right) \neq 0$ are:

$$
\iota\left(\Lambda^{1}\right), \iota\left(\Lambda^{2}\right), \iota\left(S_{(2,1)}\right), \kappa\left(\operatorname{iso}_{(x, 0)}\right), \kappa\left(\operatorname{iso}_{(x, 1)}\right), R_{H_{0}}, R_{H_{1}}, S_{H_{1}},
$$

where $R_{H_{0}}, R_{H_{1}}$ and $S_{H_{1}}$ are the simple functors introduced in Corollary 1.7. 
3.3.4. Extension groups in $\mathcal{F}_{\text {quad }}$. By Theorems 1.4 and 1.5 we obtain an exact, fully faithful functor $\prod_{V \in \mathcal{S}} \mathbb{F}_{2}[O(V)]$-mod $\stackrel{\widetilde{\kappa}}{\rightarrow} \mathcal{F}_{\text {quad }}$, where $\mathcal{S}$ is a set of representatives of isometry classes of quadratic spaces (possibly degenerate). Consequently, for any two $\mathbb{F}_{2}[O(V)]$-modules $M$ and $N$, this functor induces a morphism of extension groups

$$
\operatorname{Ext}_{\mathbb{F}_{2}[O(V)] \text {-mod }}^{*}(M, N) \stackrel{(\widetilde{\kappa})_{*}}{\longrightarrow} \operatorname{Ext}_{\mathcal{F}_{\text {quad }}}^{*}(\widetilde{\kappa}(M), \widetilde{\kappa}(N)) .
$$

We have the following proposition.

Proposition 3.32. For $V \in\left\{H_{0}, H_{1}\right\}$, the morphism $(\widetilde{\kappa})_{*}$ is an isomorphism.

The proof of this proposition relies on the following lemma.

Lemma 3.33. For $V \in\left\{H_{0}, H_{1}\right\}$, if $P$ is a finite projective $\mathbb{F}_{2}[O(V)]$ module, then $\widetilde{\kappa}(P)$ is projective in $\mathcal{F}_{\text {quad }}$.

Proof. If $P$ is a finite projective $\mathbb{F}_{2}[O(V)]$-module, then there exists an $\mathbb{F}_{2}[O(V)]$-module $Q$ such that $P \oplus Q \simeq \mathbb{F}_{2}[O(V)]^{\oplus N}$. The exactness of $\kappa$ yields $\widetilde{\kappa}(P \oplus Q) \simeq \widetilde{\kappa}(P) \oplus \widetilde{\kappa}(Q)$. Since $\widetilde{\kappa}\left(\mathbb{F}_{2}[O(V)]\right)=\kappa\left(\right.$ iso $\left._{V}\right)$ and the functors $\kappa\left(\right.$ iso $\left._{H_{0}}\right)$ and $\kappa\left(\right.$ iso $\left._{H_{1}}\right)$ are projective, so is $\widetilde{\kappa}(P)$ by Proposition 3.27.

Proof of Proposition 3.32. Let $M$ and $N$ be $\mathbb{F}_{2}[O(V)]$-modules for $V \in$ $\left\{H_{0}, H_{1}\right\}$ and $P_{\bullet} \rightarrow M$ be a projective resolution of $M$. Lemma $3.33 \mathrm{im}-$ plies that $\widetilde{\kappa}\left(P_{\bullet}\right)$ is a projective resolution of $\widetilde{\kappa}(M)$. The functor $\widetilde{\kappa}$ induces a morphism of cochain complexes

$$
\operatorname{Hom}_{\mathbb{F}_{2}[O(V)] \text {-mod }}\left(P_{\bullet}, N\right) \rightarrow \operatorname{Hom}_{\mathcal{F}_{\text {quad }}}\left(\widetilde{\kappa}\left(P_{\bullet}\right), \widetilde{\kappa}(N)\right)
$$

which induces the morphism $(\widetilde{\kappa})_{*}$ in cohomology. Since the functor $\widetilde{\kappa}$ is fully faithful, the previous morphism is an isomorphism and so induces an isomorphism in cohomology.

We deduce the following corollary:

Corollary 3.34. For any natural number n, we have

$$
\operatorname{Ext}_{\mathcal{F}_{\text {quad }}}^{n}\left(R_{H_{0}}, R_{H_{0}}\right) \simeq \mathbb{F}_{2} \text { and } \operatorname{Ext}_{\mathcal{F}_{\text {quad }}}^{n}\left(R_{H_{1}}, R_{H_{1}}\right) \simeq \mathbb{F}_{2},
$$
where $R_{H_{0}}$ and $R_{H_{1}}$ are the simple functors introduced in Corollary 1.7.

Proof. Let $\varepsilon \in\{0,1\}$. For $V=H_{\varepsilon}$, by Corollary $1.7(1)$, we have $\widetilde{\kappa}\left(\mathbb{F}_{2}\right)$ $=R_{H_{\epsilon}}$. So, applying Proposition 3.32 to $M=N=\mathbb{F}_{2}$ we obtain

$$
\operatorname{Ext}_{\mathcal{F}_{\text {quad }}}^{*}\left(R_{H_{\epsilon}}, R_{H_{\epsilon}}\right) \simeq \operatorname{Ext}_{\mathbb{F}_{2}\left[O\left(H_{\epsilon}\right)\right] \text {-mod }}^{*}\left(\mathbb{F}_{2}, \mathbb{F}_{2}\right)=H^{*}\left(O\left(H_{\epsilon}\right), \mathbb{F}_{2}\right) \text {. }
$$

Since $O\left(H_{0}\right) \simeq \mathfrak{S}_{2} \simeq C_{2}$ and $O\left(H_{1}\right) \simeq \mathfrak{S}_{3} \simeq G L_{2}\left(\mathbb{F}_{2}\right)$ we know by classical results of cohomology of groups that

$$
H^{n}\left(O\left(H_{0}\right), \mathbb{F}_{2}\right)=H^{n}\left(O\left(H_{1}\right), \mathbb{F}_{2}\right)=\mathbb{F}_{2} .
$$


REMARK 3.35. This corollary exhibits an important difference between the categories $\mathcal{F}$ and $\mathcal{F}_{\text {quad }}$; recall that $\operatorname{Ext}_{\mathcal{F}}^{1}(S, S)=0$ for all simple objects $S$ of $\mathcal{F}$ (see [8]).

4. Application: the polynomial functors in $\mathcal{F}_{\text {quad }}$. In this section, we generalize the notion of polynomial functor to the category $\mathcal{F}_{\text {quad }}$, and we prove, by induction, that these polynomial functors are in the image of the functor $\iota: \mathcal{F} \rightarrow \mathcal{F}_{\text {quad }}$.

4.1. Definition of polynomial functors in $\mathcal{F}_{\text {quad }}$

4.1.1. The difference functors in $\mathcal{F}_{\text {quad }}$. We define the difference functors in $\mathcal{F}_{\text {quad }}$ which generalize the notion of difference functor in $\mathcal{F}$. Recall that, according to [9], the difference functor $\Delta: \mathcal{F} \rightarrow \mathcal{F}$ is given by

$$
\Delta F(V):=\operatorname{Ker}\left(F\left(V \oplus \mathbb{F}_{2}\right) \stackrel{F(p)}{\longrightarrow} F(V)\right),
$$

for $F$ an object in $\mathcal{F}, V$ an object in $\mathcal{E}^{f}$ and $p: V \oplus \mathbb{F}_{2} \rightarrow V$ the projection.

Definition 4.1. The difference functors $\Delta_{H_{0}}: \mathcal{F}_{\text {quad }} \rightarrow \mathcal{F}_{\text {quad }}$ and $\Delta_{H_{1}}: \mathcal{F}_{\text {quad }} \rightarrow \mathcal{F}_{\text {quad }}$ are defined by

$$
\begin{aligned}
& \Delta_{H_{0}} F(V):=\operatorname{Ker}\left(F\left(V \perp H_{0}\right) \stackrel{F\left(T_{0}\right)}{\longrightarrow} F(V)\right), \\
& \Delta_{H_{1}} F(V):=\operatorname{Ker}\left(F\left(V \perp H_{1}\right) \stackrel{F\left(T_{1}\right)}{\longrightarrow} F(V)\right),
\end{aligned}
$$

for $F$ an object in $\mathcal{F}_{\text {quad }}, V$ an object in $\mathcal{T}_{q}$, and $T_{i}=\left[V \perp H_{i} \stackrel{\text { Id }}{\longrightarrow} V \perp H_{i}\right.$ $\left.\stackrel{i_{V}}{\longleftarrow} V\right]$ for $i \in\{0,1\}$.

Lemma 4.2. The functors $\Delta_{H_{0}}$ and $\Delta_{H_{1}}$ are exact.

4.1.2. Definition of polynomial functors. Let us first recall the definition of polynomial functors in $\mathcal{F}([9])$. An object $F$ of $\mathcal{F}$ is a polynomial functor of degree 0 if and only if $\Delta F=0$, and, for an integer $d, F$ is polynomial of degree at most $d+1$ if and only if $\Delta F$ is polynomial of degree at most $d$.

Definition 4.3. Let $F$ be an object in $\mathcal{F}_{\text {quad }}$.

(1) $F$ is polynomial of degree 0 if and only if $\Delta_{H_{0}} F=\Delta_{H_{1}} F=0$;

(2) for an integer $d, F$ is polynomial of degree at most $d+1$ if and only if $\Delta_{H_{0}} F$ and $\Delta_{H_{1}} F$ are polynomial of degree at most $d$.

The following proposition allows us to simplify the definition of a polynomial functor of degree 0 .

Lemma 4.4. Let $F$ be an object in $\mathcal{F}_{\text {quad }}$. The functor $\Delta_{H_{0}} F$ is zero if and only if $\Delta_{H_{1}} F$ is zero. 
Proof. If $\Delta_{H_{0}} F=0$, then, for all objects $V$ of $\mathcal{T}_{q}$, we have $F(V) \simeq$ $F\left(V \perp H_{0}\right)$. Consequently,

$$
F(V) \simeq F\left(V \perp H_{0}\right) \simeq F\left(V \perp H_{0} \perp H_{0}\right) \simeq F\left(V \perp H_{1} \perp H_{1}\right),
$$

where the last isomorphism is obtained from the isomorphism $H_{0} \perp H_{0} \simeq$ $H_{1} \perp H_{1}$, recalled in Section 1. From the existence of morphisms $F(V) \hookrightarrow$ $F\left(V \perp H_{1}\right)$ and $F\left(V \perp H_{1}\right) \hookrightarrow F\left(V \perp H_{1} \perp H_{1}\right)$, induced by inclusions, and from $F(V) \simeq F\left(V \perp H_{1} \perp H_{1}\right)$, we deduce that

$$
F(V) \simeq F\left(V \perp H_{1}\right) \simeq F\left(V \perp H_{1} \perp H_{1}\right) .
$$

Therefore $\Delta_{H_{1}} F=0$.

The proof of the converse is similar.

4.2. Study of polynomial functors in $\mathcal{F}_{\text {quad }}$. The aim of this section is to prove the following result:

THEOREM 4.5. The polynomial functors in $\mathcal{F}_{\text {quad }}$ are in the image of the functor $\iota: \mathcal{F} \rightarrow \mathcal{F}_{\text {quad }}$.

We will prove this theorem by induction on the degree of the polynomial functors.

4.2.1. Polynomial functors of degree zero in $\mathcal{F}_{\text {quad }}$. In this section, we start the induction. The proof of the following result relies, in an essential way, on the classification of the simple functors $S$ in $\mathcal{F}_{\text {quad }}$ such that $S\left(H_{0}\right) \neq 0$ or $S\left(H_{1}\right) \neq 0$, obtained in Proposition 3.31.

Lemma 4.6. Let $S$ be a simple functor in $\mathcal{F}_{\text {quad. Then }} S$ is a polynomial functor of degree zero if and only if $S$ is the constant functor $\mathbb{F}_{2}$.

Proof. To prove the direct implication, we distinguish two cases.

First, suppose $S\left(H_{0}\right)=S\left(H_{1}\right)=0$. By the classification of the nondegenerate quadratic spaces over $\mathbb{F}_{2}$, if $W$ is a space of minimal dimension satisfying $S(W) \neq 0$, then there exist $\varepsilon \in\{0,1\}$ and a nondegenerate quadratic space $V$ such that $W \simeq H_{\varepsilon} \perp V$. Since $W$ is of minimal dimension, we have $S(V)=0$. This implies

$$
\Delta_{H_{\varepsilon}} S(V)=S\left(H_{\varepsilon} \perp V\right) \neq 0 .
$$

This yields the result in this case.

Now, suppose $S\left(H_{0}\right) \neq 0$ or $S\left(H_{1}\right) \neq 0$. In this case, we use the classification of the simple functors $S$ in $\mathcal{F}_{\text {quad }}$ such that $S\left(H_{0}\right) \neq 0$ or $S\left(H_{1}\right) \neq 0$, obtained in Proposition 3.31. By an explicit calculation, we find that $\Delta_{H_{0}} S$ is nonzero for all functors $S$ obtained in that classification except the constant functor $S=\mathbb{F}_{2}$.

The converse is trivial. 
4.2.2. Proof of Theorem 4.5. We need the following result where the idempotents $\left[e_{V}\right]$, obtained in Proposition 2.13, play a crucial rôle.

Proposition 4.7. Let $S$ be a nontrivial simple functor in $\mathcal{F}_{\text {quad }}$ which is not in the image of the functor $\iota: \mathcal{F} \rightarrow \mathcal{F}_{\text {quad. }}$. Then either $\Delta_{H_{0}} S$ or $\Delta_{H_{1}} S$ is not in the image of $\iota$.

Proof. Let $W$ be a nondegenerate quadratic space of minimal dimension such that $S(W) \neq 0$. We distinguish two cases.

If $\operatorname{dim}(W)=2$, then the result follows by an explicit calculation for all functors $S$ of the classification given in Proposition 3.31.

If $\operatorname{dim}(W)>2$, then there exists a nondegenerate quadratic space $V$, possibly trivial, and $\varepsilon \in\{0,1\}$ such that $W \simeq H_{0} \perp H_{\varepsilon} \perp V$. Suppose that $\Delta_{H_{0}} S$ and $\Delta_{H_{1}} S$ are in the image of $\iota$; we will prove that so is $S$. By Lemma 2.18 it is sufficient to show that $S\left(e_{W}\right) S(W) \neq 0$. By Lemma 2.15, we have $e_{W}=e_{H_{0}} \perp e_{H_{\varepsilon}} \perp e_{V}$.

Since $W$ is assumed to be a space of minimal dimension such that $S(W) \neq 0$, we have $S\left(H_{0} \perp V\right)=S\left(H_{\varepsilon} \perp V\right)=0$. This implies that

$$
\begin{aligned}
& \Delta_{H_{0}} S\left(H_{\varepsilon} \perp V\right) \simeq S(W), \\
& \Delta_{H_{\varepsilon}} S\left(H_{0} \perp V\right) \simeq S(W) .
\end{aligned}
$$

These isomorphisms are natural and, for (4.7.1), the action of $\operatorname{End}_{\mathcal{T}_{q}}\left(H_{\varepsilon} \perp V\right)$ on $\Delta_{H_{0}} S\left(H_{\varepsilon} \perp V\right)$ corresponds to the restriction of the action of $\operatorname{End}_{\mathcal{T}_{q}}(W)$ on $S(W)$. In the same way, for (4.7.2), the action of $\operatorname{End}_{\mathcal{T}_{q}}\left(H_{0} \perp V\right)$ on $\Delta_{H_{\varepsilon}} S\left(H_{0} \perp V\right)$ corresponds to the restriction of the action of $\operatorname{End}_{\mathcal{T}_{q}}(W)$ on $S(W)$. Since $\Delta_{H_{0}} S$ and $\Delta_{H_{1}} S$ are in the image of $\iota$, we deduce that

$$
\begin{aligned}
S\left(1_{H_{0}} \perp e_{H_{\varepsilon}} \perp e_{V}\right) S(W) & =\Delta_{H_{0}} S\left(e_{H_{\varepsilon}} \perp e_{V}\right) \Delta_{H_{0}} S\left(H_{\varepsilon} \perp V\right) \\
& =\Delta_{H_{0}} S\left(H_{\varepsilon} \perp V\right)=S(W),
\end{aligned}
$$

where the first equality comes from the action described previously, the second is a consequence of Lemma 2.15, and the third is given by (4.7.1). In the same way, we obtain

$$
\begin{aligned}
S\left(e_{H_{0}} \perp 1_{H_{\varepsilon}} \perp e_{V}\right) S(W) & =\Delta_{H_{\varepsilon}} S\left(e_{H_{0}} \perp e_{V}\right) \Delta_{H_{\varepsilon}} S\left(H_{0} \perp V\right) \\
& =\Delta_{H_{\varepsilon}} S\left(H_{0} \perp V\right)=S(W) .
\end{aligned}
$$

Hence $S\left(1_{H_{0}} \perp e_{H_{\varepsilon}} \perp e_{V}\right) \circ S\left(e_{H_{0}} \perp 1_{H_{\varepsilon}} \perp e_{V}\right) S(W)=S(W)$. Since $S\left(1_{H_{0}} \perp e_{H_{\varepsilon}} \perp e_{V}\right) \circ S\left(e_{H_{0}} \perp 1_{H_{\varepsilon}} \perp e_{V}\right)=S\left(e_{W}\right)$ by Lemma 2.15 , we have $S\left(e_{W}\right) S(W) \neq 0$, as required.

The proof of the theorem also relies on the following lemmas.

LEMMA 4.8.

(1) A functor $F$ in $\mathcal{F}_{\text {quad }}$ which takes values in finite vector spaces and has $\Delta_{H_{\varepsilon}} F$ finite, is finite. 
(2) A polynomial functor $F$ in $\mathcal{F}_{\text {quad }}$ which takes values in finite vector spaces, is finite.

Proof. (1) The functor

$$
\mathcal{F}_{\text {quad }} \stackrel{\gamma}{\rightarrow} \mathcal{E} \times \mathcal{F}_{\text {quad }}, \quad F \mapsto\left(F(0), \Delta_{H_{\varepsilon}} F\right),
$$

is exact and faithful by Lemma 4.2. Hence, if $\gamma(F)$ is finite then so is $F$.

(2) If $F$ takes values in finite vector spaces, so does $\Delta_{H_{\varepsilon}} F$. Consequently, we can apply (1) recursively to obtain the result.

Lemma 4.9. A finite object $F$ of $\mathcal{F}_{\text {quad }}$ whose composition factors are in the image of the functor $\iota$, is in the image of $\iota$.

Proof. This is a straightforward consequence of the thickness of the subcategory $\iota(\mathcal{F})$ in $\mathcal{F}_{\text {quad }}$, stated in Theorem 2.16.

Proof of Theorem 4.5. Since a functor in $\mathcal{F}_{\text {quad }}$ is a colimit of its subfunctors which take values in finite vector spaces, Lemma 4.8 shows that it is sufficient to prove the result for finite polynomial functors in $\mathcal{F}_{\text {quad }}$. Furthermore, since the functors $\Delta_{H_{0}}$ and $\Delta_{H_{1}}$ are exact by Lemma 4.2, it is sufficient to consider the case of a simple functor $S$. We argue by induction on the polynomial degree.

If $S$ is polynomial of degree 0 , then according to Lemma $4.2, S$ is in the image of $\iota$.

Suppose that all simple polynomial functors of $\mathcal{F}_{\text {quad }}$ and of degree $d$ are in the image of $\iota$, and consider a simple polynomial functor $S$ in $\mathcal{F}_{\text {quad }}$ such that $\operatorname{deg}(S)=d+1$. By the definition of polynomial functor in $\mathcal{F}_{\text {quad }}$ given in 4.3, the functors $\Delta_{H_{0}} S$ and $\Delta_{H_{1}} S$ are polynomial of degree $d$. Hence all composition factors of $\Delta_{H_{0}} S$ and $\Delta_{H_{1}} S$ are polynomial of degree at most $d$ and, by induction, they are in the image of $\iota$. Since $S$ is a simple functor, it is a quotient of a standard projective functor $P_{V}$. Consequently, $S$ takes its values in finite-dimensional vector spaces. Therefore, $\Delta_{H_{0}} S$ and $\Delta_{H_{1}} S$ take their values in finite-dimensional vector spaces. We deduce from Lemma 4.8 that the functors $\Delta_{H_{0}} S$ and $\Delta_{H_{1}} S$ are finite, and Lemma 4.9 shows that they are in the image of $\iota$. Consequently, by Proposition 4.7, $S$ is in the image of $\iota$.

\section{References}

[1] J. Bénabou, Introduction to bicategories, in: Reports of the Midwest Category Seminar, Springer, Berlin, 1967, 1-77.

[2] N. Bourbaki, Éléments de mathématique. Première partie: Les structures fondamentales de l'analyse. Livre II: Algèbre. Chapitre 9: Formes sesquilinéaires et formes quadratiques, Actualités Sci. Ind. 1272, Hermann, Paris, 1959. 
[3] Ch. W. Curtis and I. Reiner, Methods of Representation Theory. Vol. I, Wiley, New York, 1990 (reprint of the 1981 original).

[4] V. Franjou, E. M. Friedlander, A. Scorichenko, and A. Suslin, General linear and functor cohomology over finite fields, Ann. of Math. (2) 150 (1999), 663-728.

[5] N. J. Kuhn, Generic representations of the finite general linear groups and the Steenrod algebra. I, Amer. J. Math. 116 (1994), 327-360.

[6] - Generic representations of the finite general linear groups and the Steenrod algebra. II, K-Theory 8 (1994), 395-428.

[7] A. Pfister, Quadratic Forms with Applications to Algebraic Geometry and Topology, London Math. Soc. Lecture Note Ser. 217, Cambridge Univ. Press, Cambridge, 1995.

[8] L. Piriou and L. Schwartz, Extensions de foncteurs simples, K-Theory 15 (1998), 269-291.

[9] L. Schwartz, Unstable Modules over the Steenrod Algebra and Sullivan's Fixed Point Set Conjecture, Chicago Lectures in Mathematics, Univ. of Chicago Press, Chicago, IL, 1994.

[10] C. Vespa, La catégorie $\mathcal{F}_{\text {quad }}$ des foncteurs de Mackey généralisés pour les formes quadratiques sur $\mathbb{F}_{2}$, Ph.D. thesis, Univ. Paris 13, 2005.

[11] - Generic representations of orthogonal groups: the mixed functors, Algebr. Geom. Topology 7 (2007), 379-410.

[12] - Generic representations of orthogonal groups: the functor category $\mathcal{F}_{\text {quad }}$, J. Pure Appl. Algebra 212 (2008), 1472-1499.

Institut de Recherche Mathématique Avancée

Université Louis Pasteur

7 rue René Descartes

67084 Strasbourg Cédex, France

E-mail: vespa@math.u-strasbg.fr

Received 15 April 2007;

in revised form 11 April 2008 